\title{
$W$-WIMP Annihilation as a Source of the Fermi Bubbles
}

\author{
Luis Alfredo Anchordoqui* and Brian James Vlcek ${ }^{\dagger}$ \\ Department of Physics, \\ University of Wisconsin-Milwaukee, \\ Milwaukee, WI 53201, USA
}

(Dated: May 2013)

\begin{abstract}
The Fermi Gamma-ray Space Telescope discovered two $\gamma$-ray emitting bubble-shaped structures that extend nearly symmetrically on either side of our Galaxy and appear morphologically connected to the Galactic center. The origin of the emission is still not entirely clear. It was recently shown that the spectral shape of the emission from the Fermi bubbles is well described by an approximately $50 \mathrm{GeV}$ dark matter particle annihilating to $b \bar{b}$, with a normalization corresponding to a velocity average annihilation cross section $\left\langle\sigma_{b} v\right\rangle \approx 8 \times 10^{-27} \mathrm{~cm}^{3} / \mathrm{s}$. We study the minimal hidden sector recently introduced by Weinberg and examine to what extent its weakly interacting massive particles ( $W$-WIMPs) are capable of accommodating both the desired effective annihilation rate into quarks and the observed dark matter density.
\end{abstract}

*Electronic address: luis.anchordoqui@gmail.com

$\dagger$ Electronic address: brian.vlcek@gmail.com 


\section{INTRODUCTION}

Beyond standard model (SM) physics models to be probed at the Large Hadron Collider (LHC) often include the concept of a hidden sector, consisting of $S U(3) \times S U(2) \times U(1)$ singlet fields. Independent of any model, the standard sector and the hidden sector are coupled by interactions of gauge-invariant operators which illuminate the path for exploring structures in the hidden sector by observing phenomena in the visible standard sector. A tantalizing realization of this idea is provided by the Higgs portal, which connects the Higgs fields in the two sectors by an elementary quartic interaction [1-8]. Such a construct moves a precision study of the Higgs sector into a central position of new physics searches at the LHC. Likewise, astrophysical observations open the gates for complementary information to further test the Higgs portal hypothesis and to improve our understanding of the physics in the hidden sector.

Around the Galactic center (GC), there exists a bright and spatially extended source $\gamma$-ray emission peaking at energies of a few $\mathrm{GeV}$. The spectrum and morphology of this signal is consistent with one originating from dark matter annihilations [9-12]. Very recently, evidence of this signal has been found from regions outside of the GC [13] in the directions of the sky coincident with the Fermi bubbles: two bilateral $\gamma$-ray lobes centered at the core of the Galaxy and extending to around $50^{\circ}$ above and below the Galactic plane (i.e., $r= \pm 10 \mathrm{kpc}$, where $r$ is the distance from the GC) [14, 15]. At lower Galactic latitudes, these structures are coincident with a nonthermal microwave "haze" found in WMAP $23-$ $33 \mathrm{GHz}$ data [16] (confirmed recently by the Planck space mission [17]) and the thermal x-ray emission seen by ROSAT [18].

Far from the Galactic plane $\left(|b| \gtrsim 30^{\circ}\right)$, the observed energy-weighted $\gamma$-ray spectrum is nearly invariant with latitude and fairly flat $\left(d \Phi_{\gamma} / d E_{\gamma} \propto E_{\gamma}^{-2}\right)$ over the energy range observed by Fermi. The correlation found in the multiwavelength observations seems to indicate that the bubbles (measured in the range of $E_{\gamma} \sim 1-100 \mathrm{GeV}$ ) are produced by a population of $\mathrm{GeV}-\mathrm{TeV}$ electrons (with an approximately power-law spectrum $d \Phi_{e} / d E_{e} \propto$ $\left.E_{e}^{-3}\right)$ via inverse Compton scattering of ambient low-energy photons, as the same electrons can also simultaneously produce radio synchrotron radiations in the presence of magnetic fields $[14,19]$. The transparency of this elementary and self-consistent framework provides strong support for a leptonic origin of the high-latitude emission from the Fermi bubbles.

Conversely, at latitudes closer to the disk $\left(|b| \lesssim 20^{\circ}\right)$, the spectrum of the emission correlated with the bubbles possesses a pronounced spectral feature in $E_{\gamma}^{2} d \Phi_{\gamma} / d E_{\gamma}$ peaking at $E_{\gamma} \sim 1-4 \mathrm{GeV}$, which cannot be produced by any realistic spectrum of electrons [13]. This implies that a second (non-inverse-Compton) emission mechanism must be responsible for the bulk of the low-energy, low-latitude emission. The spectral shape of this second component is similar to the one reported from the GC. The intrinsic non-inverse-Compton emission appears spatially consistent with a luminosity per volume falling approximately

as $r^{-2.4}-r^{-2.8}$. As a consequence, the spectral feature visible in the low-latitude bubbles is most likely the extended counterpart of the GC excess, now detected out to at least $r \sim 2-3 \mathrm{kpc}$. Even though millisecond pulsars possess a spectral cutoff at approximately the required energy, these sources exhibit a spectral shape that is much too soft at sub-GeV energies to accommodate this signal [20].

The spectrum and angular distribution of the signal is broadly consistent with one predicted from $\sim 10 \mathrm{GeV}$ dark matter particles annihilating to leptons, or from $\sim 50 \mathrm{GeV}$ dark matter particles annihilating to quarks, following a distribution similar to, but slightly 
steeper than, the canonical Navarro-Frenk-White (NFW) profile. In either case, the morphology of the $\gamma$-ray signal requires a dark matter distribution that scales approximately as $\rho_{\mathrm{DM}} \propto r^{-1.2}-r^{-1.4}$; that is, the annihilation rate per volume is proportional to the square of the dark matter density. Such a dark matter distribution is in good agreement with current observational constraints [21].

For the $10 \mathrm{GeV}$ dark matter candidate, the normalization of the observed signal requires a velocity average annihilation cross section on the order of

$$
\left\langle\sigma_{\tau} v\right\rangle \sim 2 \times 10^{-27} \mathrm{~cm}^{3} / \mathrm{s}=1.7 \times 10^{-10} \mathrm{GeV}^{-2},
$$

up to overall uncertainties in the normalization of the halo profile [22]. This light mass scenario has been further invigorated by various observations reported by the DAMA/LIBRA [23], CoGeNT [24, 25], CRESST [26], and CDMS [27] collaborations, each of which report signals consistent with a dark matter particle of similar mass. These four experiments make use of different technologies, target materials, and detection strategies, but each reports results that are not compatible with known backgrounds but which can be accommodated by a light dark matter particle with a mass of about $10 \mathrm{GeV}$ and an elastic scattering cross section with nucleons of $1-2 \times 10^{-41} \mathrm{~cm}^{2}$ [28-31].

Dark matter particles can elastically scatter with nuclei in the Sun, leading to their gravitational capture and subsequent annihilation. Electrons and muons produced in such annihilations quickly lose their energy to the solar medium and produce no observable effects. Annihilations to taus, on the other hand, produce neutrinos which, for a $10 \mathrm{GeV}$, can be observed by Super-Kamiokande. For the required branching into $\tau^{+} \tau^{-}$of about $10 \%$ - as given by (1) - existing data constrain the dark matter spin-independent elastic scattering cross section with protons to be less than $4 \times 10^{-41} \mathrm{~cm}^{2}[32,33]$.

For the $50 \mathrm{GeV}$ dark matter particle, the normalization of the observed signal requires a velocity average annihilation cross section on the order of

$$
\left\langle\sigma_{b} v\right\rangle \sim 8 \times 10^{-27} \mathrm{~cm}^{3} / \mathrm{s}=6.7 \times 10^{-10} \mathrm{GeV}^{-2} .
$$

The XENON-100 Collaboration reported a 90\% C.L. bound on the elastic scattering cross section with nuclei of $\mathcal{O}\left(10^{-44} \mathrm{~cm}^{2}\right)$ [34]. A later analysis arrived at alternative conclusions allowing for a signal of two events with a favored mass of $12 \mathrm{GeV}$ and large error contour extending to about $50 \mathrm{GeV}$ [35].

It is worthwhile to point out that the bounds from the combined analysis of 10 dwarf spheroidals [36, 37], galaxy clusters [38], or diffuse $\gamma$-ray emission [39, 40] are not sensitive enough to probe the velocity average annihilation cross sections (1) and (2).

In this paper we study the minimal hidden sector of Weinberg's Higgs portal model [41], and we examine to what extent its free parameters can be adjusted to explain the lowlatitude $\gamma$-ray emission from the Fermi bubbles. The layout of the paper is as follows. In Sec. II we outline the basic setting of the model. In Sec. III we review the constraints related to experimental searches for new physics at the LHC. After that, in Sec. IV we turn our attention to the prospects for direct dark matter searches. In Sec. V we study the constraints from cosmological observations. In Sec. VI we present the main results of this work. We begin by constraining the parameter space in the Higgs sector along a correlation of the Fermi bubbles' $\gamma$-ray signal with the dark matter annihilation cross section into SM fermions. We then further constrain the parameter space by matching the thermal relic abundance of dark matter with the value inferred by cosmological observations. Lastly, in Sec. VII we explore a region of parameter space which cannot accommodate Fermi observations but remains interesting in itself. In Sec. VIII we summarize our findings. 


\section{II. $W$-WIMPs}

Weinberg's Higgs portal model is based on a broken global $U(1)$ symmetry associated with the dark matter charge $W$ : the number of weakly interacting massive particles (WIMPs) minus the number of their antiparticles. The hidden sector contains a Dirac field $\psi$ (carrying WIMP quantum number $W=+1$ ) and a complex scalar field (with $W=2$, so that its expectation value leaves an unbroken reflection symmetry $\psi \rightarrow-\psi$ ). All SM fields are assumed to have $W=0$.

The scalar potential consists of the SM component [s], the isomorphic component in the hidden sector $[h]$, and the quartic interaction coupling between the two sectors with strength $\eta_{\chi}$. The Lagrangian density for the scalar sector reads

$$
\mathscr{L}=\left|\partial \Phi_{h}\right|^{2}+\left|\partial \Phi_{s}\right|^{2}+\mu_{h}^{2}\left|\Phi_{h}\right|^{2}-\lambda_{h}\left|\Phi_{h}\right|^{4}+\mu_{s}^{2}\left|\Phi_{s}\right|^{2}-\lambda_{s}\left|\Phi_{s}\right|^{4}-\eta_{\chi}\left|\Phi_{h}\right|^{2}\left|\Phi_{s}\right|^{2},
$$

where $\Phi_{s}$ is the SM scalar doublet and $\Phi_{h}$ is a complex scalar field. We separate a massless Goldstone boson field $\alpha(x)$ and a massive radial field $r(x)$ by defining

$$
\Phi_{h}(x)=\frac{1}{\sqrt{2}} r(x) e^{i 2 \alpha(x)},
$$

where $r(x)$ and $\alpha(x)$ are real, with the phase of $\Phi_{h}(x)$ adjusted to make the vacuum expectation value $(\mathrm{VEV})$ of $\alpha(x)$ zero. The $S U(2) \times U(1)$ symmetry of the SM is (of course) broken by a nonvanishing VEV of the neutral component $\phi$ of the scalar doublet,

$$
\Phi_{s}=\frac{1}{\sqrt{2}}\left(\begin{array}{c}
G^{ \pm} \\
v_{\phi}+\phi^{\prime}+i G^{0}
\end{array}\right),
$$

where $v_{\phi} \simeq 246 \mathrm{GeV}$. The $G$ fields are the familiar Goldstone bosons, which are eaten by the vector bosons (i.e. the $G^{ \pm}$become the longitudinal components of the charged $W$ boson and $G^{0}$ becomes the longitudinal component of the $Z$ boson). In terms of real fields the Lagrangian density (3) takes the form

$$
\mathscr{L}=\frac{1}{2} \partial r^{2}+\frac{1}{2} \partial \phi^{2}+2 r^{2} \partial \alpha^{2}+\frac{\mu_{h}^{2}}{2} r^{2}-\frac{\lambda_{h}}{4} r^{4}+\mu_{s}^{2}|\phi|^{2}-\lambda_{s}|\phi|^{4}-\frac{\eta_{\chi}}{2} r^{2}|\phi|^{2} .
$$

The $U(1)$ symmetry of $W$ conservation is also broken and $r$ gets a VEV

$$
r(x)=v_{r}+r^{\prime}(x)
$$

with $v_{r}$ real and non-negative.

We demand the scalar potential obtains its minimum value at

$$
\mathcal{V}=-\frac{\mu_{h}^{2}}{2} v_{r}^{2}+\frac{\lambda_{h}}{4} v_{r}^{4}-\frac{\mu_{s}^{2}}{2} v_{\phi}^{2}+\frac{\lambda_{s}}{4} v_{\phi}^{4}+\frac{\eta_{\chi}}{4} v_{r}^{2} v_{\phi}^{2}
$$

Physically, the most interesting solutions to the minimization of (8),

$$
\partial_{v_{r}} \mathcal{V}=-\mu_{h}^{2} v_{r}+\lambda_{h} v_{r}^{3}+\frac{\eta_{\chi}}{2} v_{r} v_{\phi}^{2}=0
$$

and

$$
\partial_{v_{\phi}} \mathcal{V}=-\mu_{s}^{2} v_{\phi}+\lambda_{s} v_{\phi}^{3}+\frac{\eta_{\chi}}{2} v_{r}^{2} v_{\phi}=0
$$


are obtained for $v_{r}$ and $v_{\phi}$ both nonvanishing

$$
v_{\phi}^{2}=\frac{1}{\lambda_{s}}\left(\mu_{s}^{2}-\frac{\eta_{\chi} v_{r}^{2}}{2}\right)
$$

and

$$
v_{r}^{2}=\frac{1}{\lambda_{h}}\left(\mu_{h}^{2}-\frac{\eta_{\chi} v_{\phi}^{2}}{2}\right),
$$

respectively. To compute the scalar masses, we must expand the potential around the minima

$$
\begin{aligned}
\mathscr{L} & =\frac{1}{2}\left(\partial r^{\prime}\right)^{2} \\
& +2 v_{r}^{2} \partial \alpha^{2}+4 v_{r} r^{\prime} \partial \alpha^{2}+2 r^{\prime 2} \partial \alpha^{2} \\
& -\lambda_{h} v_{r}^{2} r^{\prime 2}-\lambda_{s} v_{\phi}^{2} \phi^{\prime 2}-\eta_{\chi} v_{r} v_{\phi} r^{\prime} \phi^{\prime}+\cdots,
\end{aligned}
$$

where the dots indicate 3-point and 4-point interactions, as well as the SM interactions. There is a mixing term present for $r^{\prime}$ and $\phi^{\prime}$. We find the fields of definite mass by diagonalizing the mass matrix for $r^{\prime}$ and $\phi^{\prime}$. We denote by $H$ and $h$ the scalar fields of definite masses, $m_{H}=125 \mathrm{GeV}$ and $m_{h}$, respectively. After a bit of algebra, the explicit expressions for the scalar mass eigenvalues and eigenvectors are given by

$$
m_{h}^{2}=\lambda_{h} v_{r}^{2}+\lambda_{s} v_{\phi}^{2}-\sqrt{\left(\lambda_{s} v_{\phi}^{2}-\lambda_{h} v_{r}^{2}\right)^{2}+\left(\eta_{\chi} v_{r} v_{\phi}\right)^{2}}
$$

and

$$
m_{H}^{2}=\lambda_{h} v_{r}^{2}+\lambda_{s} v_{\phi}^{2}+\sqrt{\left(\lambda_{s} v_{\phi}^{2}-\lambda_{h} v_{r}^{2}\right)^{2}+\left(\eta_{\chi} v_{r} v_{\phi}\right)^{2}}
$$

with

$$
\left(\begin{array}{l}
h \\
H
\end{array}\right)=\left(\begin{array}{rr}
\cos \chi & -\sin \chi \\
\sin \chi & \cos \chi
\end{array}\right)\left(\begin{array}{l}
r^{\prime} \\
\phi^{\prime}
\end{array}\right)
$$

where $\chi \in[-\pi / 2, \pi / 2]$ also fulfills

$$
\sin 2 \chi=\frac{\eta_{\chi} v_{\phi} v_{r}}{\sqrt{\left(\lambda_{s} v_{\phi}^{2}-\lambda_{h} v_{r}^{2}\right)^{2}+\left(\eta_{\chi} v_{r} v_{\phi}\right)^{2}}}=\frac{2 \eta_{\chi} v_{\phi} v_{r}}{m_{H}^{2}-m_{h}^{2}},
$$

and

$$
\cos 2 \chi=\frac{\lambda_{s} v_{\phi}^{2}-\lambda_{h} v_{r}^{2}}{\sqrt{\left(\lambda_{s} v_{\phi}^{2}-\lambda_{h} v_{r}^{2}\right)^{2}+\left(\eta_{\chi} v_{r} v_{\phi}\right)^{2}}}
$$

yielding

$$
\tan 2 \chi=\frac{\eta_{\chi} v_{r} v_{\phi}}{\lambda_{s} v_{\phi}^{2}-\lambda_{h} v_{r}^{2}}
$$

The Goldstone boson in (13) has to be be renormalized so that it resumes the standard canonical form. This is achieved through scaling $\alpha \rightarrow \alpha^{\prime}=2 v_{r} \alpha$, videlicet,

$$
2 v_{r}^{2} \partial \alpha^{2}+4 v_{r} r^{\prime} \partial \alpha^{2}+2 r^{\prime 2} \partial \alpha^{2} \rightarrow \frac{1}{2} \partial \alpha^{\prime 2}+\frac{1}{v_{r}} r^{\prime} \partial \alpha^{\prime 2}+\frac{1}{2 v_{r}^{2}} r^{\prime 2} \partial \alpha^{\prime 2} .
$$


Adding in the dark matter sector requires at least one Dirac field

$$
\mathscr{L}_{\psi}=i \bar{\psi} \gamma \cdot \partial \psi-m_{\psi} \bar{\psi} \psi-\frac{f}{\sqrt{2}} \bar{\psi}^{c} \psi \Phi_{h}^{\dagger}-\frac{f^{*}}{\sqrt{2}} \bar{\psi} \psi^{c} \Phi_{h}
$$

We assign $\psi$ a charge $W=1$, so that the Lagrangian is invariant under the global transformation $e^{i W \alpha}$. Treating the transformation as local allows us to express $\psi$ as

$$
\psi(x)=\psi^{\prime}(x) e^{i \alpha(x)}
$$

We can now rewrite $(21)$ in terms of $\psi^{\prime}, \alpha$, and $r$

$$
\mathscr{L}_{\psi}=i \bar{\psi}^{\prime} \gamma \cdot \partial \psi^{\prime}-\left(\bar{\psi}^{\prime} \gamma \psi^{\prime}\right) \cdot \partial \alpha-m_{\psi} \bar{\psi}^{\prime} \psi^{\prime}-\frac{f}{2} \bar{\psi}^{\prime c} \psi^{\prime} r-\frac{f}{2} \bar{\psi}^{\prime} \psi^{\prime c} r
$$

where we have taken $f$ to be real. Once $r$ achieves a VEV we can expand the dark matter sector to get

$$
\begin{aligned}
\mathscr{L}_{\psi} & =\frac{i}{2}\left(\bar{\psi}^{\prime} \gamma \cdot \partial \psi^{\prime}+\bar{\psi}^{c} \gamma \cdot \partial \psi^{c^{\prime}}\right) \\
& -\frac{m_{\psi}}{2}\left(\bar{\psi}^{\prime} \psi^{\prime}+\bar{\psi}^{c} \psi^{\prime c}\right)-\frac{f v_{r}}{2} \bar{\psi}^{\prime} \psi^{\prime}-\frac{f v_{r}}{2} \bar{\psi}^{\prime} \psi^{\prime c} \\
& -\frac{1}{2}\left(\bar{\psi}^{\prime} \gamma \psi^{\prime}-\bar{\psi}^{\prime} \gamma \psi^{\prime c}\right) \cdot \partial \alpha \\
& -\frac{f}{2} r^{\prime}\left(\bar{\psi}^{c} \psi^{\prime}+\bar{\psi}^{\prime} \psi^{\prime c}\right) .
\end{aligned}
$$

Note that we have made the Lagrangian explicitly symmetric via relations like

$$
\begin{aligned}
\psi^{c} & =C \bar{\psi}^{T} \\
\bar{\psi}^{c} \psi^{c} & =\left(-\psi^{T} C^{-1} C \bar{\psi}^{T}\right)=\bar{\psi} \psi \\
\bar{\psi}_{c} \gamma \cdot \partial \psi_{c} & =-\psi^{T} C^{-1} \gamma C \cdot \partial \bar{\psi}^{T} \\
& =\psi^{T} \gamma^{T} \cdot \partial \bar{\psi}^{T}=-(\partial \bar{\psi} \cdot \gamma \psi) \rightarrow \bar{\psi} \gamma \cdot \partial \psi
\end{aligned}
$$

In (26) we used the Grassman nature of the spinor fields; in the second line of (27) we used integration by parts to transfer the derivative onto the $\psi$ field. Similar results can be found for the other expressions.

Diagonalization of the $\psi^{\prime}$ mass matrix generates the mass eigenvalues,

$$
m_{ \pm}=m_{\psi} \pm f v_{r}
$$

for the two mass eigenstates

$$
\psi_{-}=\frac{i}{\sqrt{2}}\left(\psi^{\prime c}-\psi^{\prime}\right) \quad \text { and } \quad \psi_{+}=\frac{1}{\sqrt{2}}\left(\psi^{\prime c}+\psi^{\prime}\right) .
$$

In this basis, the act of charge conjugation on $\psi_{ \pm}$results in

$$
\psi_{ \pm}^{c}=\psi_{ \pm}
$$


This tells us that the fields $\psi_{ \pm}$are Majorana fermions. The Lagrangian is found to be

$$
\begin{aligned}
\mathscr{L}_{\psi} & =\frac{i}{2} \bar{\psi}_{+} \gamma \cdot \partial \psi_{+}+\frac{i}{2} \bar{\psi}_{-} \gamma \cdot \partial \psi_{-}-\frac{1}{2} m_{+} \bar{\psi}_{+} \psi_{+}-\frac{1}{2} m_{-} \bar{\psi}_{-} \psi_{-}, \\
& -\frac{i}{4 v_{r}}\left(\bar{\psi}_{+} \gamma \psi_{-}-\bar{\psi}_{-} \gamma \psi_{+}\right) \cdot \partial \alpha^{\prime} \\
& -\frac{f}{2} r^{\prime}\left(\bar{\psi}_{+} \psi_{+}-\bar{\psi}_{-} \psi_{-}\right) .
\end{aligned}
$$

We must now put $r^{\prime}$ into its massive field representation, for which the interactions of interest are

$$
-\frac{f \sin \chi}{2} H\left(\bar{\psi}_{+} \psi_{+}-\bar{\psi}_{-} \psi_{-}\right)-\frac{f \cos \chi}{2} h\left(\bar{\psi}_{+} \psi_{+}-\bar{\psi}_{-} \psi_{-}\right)
$$

This leads to 3-point interactions between the $W$-WIMPs and the Higgs boson of the SM.

In summary, instead of one Dirac $W$-WIMP, there are two Majorana $W$-WIMPs of different masses. However, the heavier $W$-WIMP will decay into the lighter one by emitting a Goldstone boson, while the lighter one is kept stable by an unbroken reflection symmetry. Therefore in this model we can expect that the Universe today will contain only one type of Majorana $W$-WIMP, the lighter one $w$, with mass $m_{w}$ equal to the smaller of $m_{ \pm}$. Throughout, $\Delta m=\left|m_{+}-m_{-}\right|=2\left|f v_{r}\right|$ denotes the mass splitting of the $W$-WIMP states. (The most common variables used in this article are summarized in Table I.)

A cautionary note is worth taking on board at this juncture. It has long been known that the spontaneous breaking of a global $U(1)$ symmetry has several disconnected and degenerate vacua (the phase of the vacuum expectation value $\left\langle 0\left|\Phi_{h}\right| 0\right\rangle$ can be different in different regions of space, and actually we expect it to be different in casually disconnected regions), leading to catastrophic domain-wall structures in the early Universe [42, 43]. In the spirit of [42], it may be possible to introduce a small explicit breaking of the symmetry, such that the domain walls disappear before dominating the matter density of the Universe, while leaving (pseudo-)Goldstone bosons and the same dark matter phenomenology. ${ }^{1}$

The absence of new physics signals at the LHC place constraints on the model. We discuss this next.

\section{CONSTRAINTS FROM COLLIDER EXPERIMENTS}

The recent discovery $[49,50]$ of a new particle with properties consistent with the SM Higgs boson is without any doubt the most compelling news from the LHC. With the measurements in various channels, a comprehensive study of the properties of the Higgs-like state becomes possible and has the potential for revealing whether or not the Higgs sector is as simple as envisioned in the SM. Since invisible decays reduce the branching fraction to the (visible) SM final states, it is to be expected that $\mathcal{B}(H \rightarrow$ invisible) is strongly constrained. Indeed $\mathcal{B}(H \rightarrow$ invisible $)$ is known to be less than about $19 \%$ at $95 \%$ C.L. [51-54]. Thus, the mixing of the SM with the hidden sector must be weak. Note that for $\eta_{\chi} \ll 1$ the relations between masses and angles then become

$$
m_{h}^{2} \approx 2 \lambda_{h} v_{r}^{2}, \quad m_{H}^{2} \approx 2 \lambda_{s} v_{\phi}^{2}, \quad \tan 2 \chi \approx \frac{2 \eta_{\chi} v_{r} v_{\phi}}{m_{H}^{2}-m_{h}^{2}},
$$

\footnotetext{
${ }^{1}$ Other approaches, if exceedingly fine-tuned, may offer alternative solutions [44-48].
} 
TABLE I: Definition of most common variables.

\begin{tabular}{ll}
\hline \hline$\Phi_{s}$ & SM scalar doublet \\
\hline$\Phi_{h}$ & Complex scalar field \\
\hline$\phi$ & Neutral component of the scalar doublet \\
\hline$r$ & Massive radial field \\
\hline$\alpha$ & Goldstone boson \\
\hline$v_{\phi}$ & Vacuum expectation value of $\phi$ \\
\hline$v_{r}$ & Vacuum expectation value of $r$ \\
\hline$H$ & SM Higgs boson \\
\hline$h$ & Hidden scalar \\
\hline$\eta_{\chi}$ & Quartic interaction coupling between SM and hidden sectors \\
\hline$\chi$ & $H$ - $h$ mixing angle \\
\hline$w$ & Lightest $W$-WIMP \\
\hline$\Delta m$ & $W$-WIMP mass splitting \\
\hline$f$ & Coupling between hidden Majorana fermions and complex scalar field \\
\hline \hline
\end{tabular}

where we have assumed $\lambda_{s} v_{\phi}^{2}>\lambda_{h} v_{r}^{2}$. For a Higgs width of about $4 \mathrm{MeV}$, the partial width for decay into unobserved particles is found to be

$$
\Gamma_{H \rightarrow \text { invisible }}<0.8 \mathrm{MeV} \text {. }
$$

The phenomenology of a Higgs portal to the hidden sector depends on whether the SM Higgs particle is lighter or heavier than the new companion. In this study we take $m_{H}>m_{h}$.

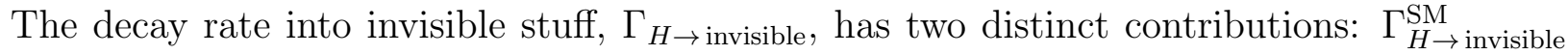
and $\Gamma_{H \rightarrow \text { hidden. }}$ The former is dominated by $H \rightarrow 2 Z \rightarrow 4 \nu$, with an invisible $Z$ branching ratio of $4 \%$. The $4 \nu$ rate can be predicted from observed decays $H \rightarrow 2 Z \rightarrow 4 l$. For the sake of simplicity, hereafter we will omit the contribution of $\Gamma_{H \rightarrow \text { invisible }}^{\mathrm{SM}}$ Unless expressly stated otherwise herein, we assume $m_{w}+\Delta m>m_{H} / 2$ and thus $H$ decays (invisibly) into the hidden sector via three channels: $H \rightarrow 2 \alpha^{\prime}, H \rightarrow 2 h$, and $H \rightarrow 2 w$. From the event rates for visible Higgs production and decay channels, we could derive upper bounds on non-SM admixtures in the wave function of the Higgs boson and on the new three invisible decay channels. To this end we now compute the decay rates for these three processes.

\section{A. $\Gamma_{H \rightarrow 2 \alpha^{\prime}}$}

Substituting in (20) $r^{\prime}$ by the field of definite mass, $r^{\prime}=h \cos \chi+H \sin \chi$, we can write the Higgs-Goldstone boson interaction term as

$$
\frac{1}{v_{r}} r^{\prime} \partial \alpha^{\prime 2} \rightarrow \frac{\sin \chi}{v_{r}} H\left(\partial \alpha^{\prime}\right)^{2}+\frac{\cos \chi}{v_{r}} h\left(\partial \alpha^{\prime}\right)^{2}
$$

Using (35) we write the Feynman rule for interactions of the type $H, \alpha^{\prime}, \alpha^{\prime}$ as

$$
-i \frac{2 \sin \chi}{v_{r}} k \cdot k^{\prime}
$$


where $k\left(k^{\prime}\right)$ is the 4-momentum of the incoming (outgoing) $\alpha^{\prime}$ particle, and the factor of 2 is a symmetry factor, as one can exchange incoming-outgoing $\alpha^{\prime}$ twice. From this 3-point interaction we can calculate the decay width of the SM Higgs $H$ into 2 Goldstone bosons $\alpha^{\prime}$. In the rest frame of the Higgs, the differential decay probability per unit time is given by

$$
d \Gamma_{H \rightarrow 2 \alpha^{\prime}}=\frac{1}{2 m_{H}}\left(\frac{2 \sin \chi}{v_{r}} k_{1} \cdot k_{2}\right)^{2} d \mathcal{Q},
$$

where

$$
\begin{aligned}
d \mathcal{Q} & =\frac{1}{2 !} \frac{d^{3} k_{1}}{(2 \pi)^{3} 2 k_{1}} \frac{d^{3} k_{2}}{(2 \pi)^{3} 2 k_{2}}(2 \pi)^{4} \delta\left(m_{H}-k_{1}-k_{2}\right) \delta^{(3)}\left(\mathbf{k}_{1}+\mathbf{k}_{2}\right) \\
& =\left.\frac{1}{16} \frac{d \Omega_{k_{1}}}{(2 \pi)^{2}}\right|_{k_{1}=m_{H} / 2}
\end{aligned}
$$

is the phase space for a two-body final state (the factor of $1 / 2$ ! is included because of identical particles in the final state). After some algebra (37) can be rewritten as

$$
d \Gamma_{H \rightarrow 2 \alpha^{\prime}}=\frac{d \Omega_{k_{1}}}{128 \pi^{2} m_{H}}\left[\frac{2 \sin \chi}{v_{r}} 2\left(\frac{m_{H}}{2}\right)^{2}\right]^{2} .
$$

The partial decay width can now be expressed as

$$
\Gamma_{H \rightarrow 2 \alpha^{\prime}}=\frac{1}{32 \pi}\left(\frac{\sin \chi}{v_{r}}\right)^{2} m_{H}^{3} .
$$

For $m_{H} \gg m_{h}$ and $m_{H}^{2} \gg 2 \eta_{\chi} v_{r} v_{\phi}$, we can use the small angle approximation

$$
\sin \chi \approx \chi=\eta_{\chi} v_{r} v_{\phi} /\left(m_{H}^{2}-m_{h}^{2}\right) .
$$

In this very good approximation the decay width becomes

$$
\Gamma_{H \rightarrow 2 \alpha^{\prime}}=\frac{1}{32 \pi}\left(\frac{\eta_{\chi} v_{\phi}}{m_{H}^{2}-m_{h}^{2}}\right)^{2} m_{H}^{3} .
$$

\section{B. $\Gamma_{H \rightarrow 2 h}$}

We begin by expanding the scalar potential $\mathcal{V}$ around the VEVs of $r$ and $\phi$ after which we diagonalize the mass matrix. Together this requires that we expand around the fields

$$
\begin{aligned}
& r(x)=v_{r}+h \cos \chi+H \sin \chi, \\
& \phi(x)=v_{\phi}+H \cos \chi-h \sin \chi,
\end{aligned}
$$


which puts $\mathcal{V}$ in the form

$$
\begin{aligned}
\mathcal{V} & =\frac{1}{2} m_{H}^{2} H^{2}+\frac{1}{2} m_{h}^{2} h^{2} \\
& -\frac{1}{16}\left(\eta_{\chi}+3\left(\lambda_{h}+\lambda_{s}\right)+3\left(\eta_{\chi}-\lambda_{h}-\lambda_{s}\right) \cos 4 \chi\right) H^{2} h^{2} \\
& -\frac{1}{4} v_{\phi} \cos \chi\left[6 \lambda_{s}-\eta_{\chi}+3\left(\eta_{\chi}-2 \lambda_{s}\right) \cos 2 \chi\right] H h^{2} \\
& -\frac{1}{4} v_{r} \sin \chi\left[6 \lambda_{h}-\eta_{\chi}-3\left(\eta_{\chi}-2 \lambda_{h}\right) \cos 2 \chi\right] H h^{2} \\
& -\frac{1}{4}\left(\lambda_{s} \cos ^{4} \chi+\eta_{\chi} \cos ^{2} \chi \sin ^{2} \chi+\lambda_{h} \sin ^{4} \chi\right) H^{4} \\
& -\frac{1}{4}\left(\lambda_{h} \cos ^{4} \chi+\eta_{\chi} \cos ^{2} \chi \sin ^{2} \chi+\lambda_{s} \sin ^{4} \chi\right) h^{4} \\
& +\frac{1}{2}\left(v_{\phi} \sin \chi\left(\eta_{\chi} \cos ^{2} \chi+2 \lambda_{s} \sin ^{2} \chi\right)-v_{r}\left(2 \lambda_{h} \cos ^{3} \chi+\eta_{\chi} \cos \chi \sin ^{2} \chi\right)\right) h^{3} \\
& -\frac{1}{2}\left(v_{r} \sin \chi\left(\eta_{\chi} \cos ^{2} \chi+2 \lambda_{h} \sin ^{2} \chi\right)+v_{\phi}\left(2 \lambda_{s} \cos ^{3} \chi+\eta_{\chi} \cos \chi \sin ^{2} \chi\right)\right) H^{3} \\
& -\frac{1}{4}\left(\lambda_{h}-\lambda_{s}+\left(\lambda_{s}+\lambda_{h}-\eta_{\chi}\right) \cos 2 \chi\right) \sin ^{2} 2 H^{3} \\
& +\frac{1}{4}\left(\lambda_{s}-\lambda_{h}+\left(\lambda_{s}+\lambda_{h}-\eta_{\chi}\right) \cos 2 \chi\right) \sin ^{3} \chi H^{3} h \\
& +\frac{1}{2} v_{\phi} \sin \chi\left[2\left(3 \lambda_{s}-\eta_{\chi}\right) \cos ^{2} \chi+\eta_{\chi} \sin ^{2} \chi\right] H^{2} h \\
& +\frac{1}{2} v_{r}\left(\eta_{\chi} \sin \chi \sin _{2} \chi-6 \lambda_{h} \cos \chi \sin ^{2} \chi-\eta_{\chi} \cos ^{3} \chi\right) H^{2} h .
\end{aligned}
$$

Since $\chi<1$ we first expand the potential around $\chi=0$, and then using (17) we further expand around $\eta_{\chi}=0$ retaining only the terms first order in $\eta_{\chi}$; this results in

$$
\begin{aligned}
\mathcal{V} & \approx \frac{1}{2} m_{H}^{2} H^{2}+\frac{1}{2} m_{h}^{2} h^{2} \\
& -\frac{\eta_{\chi}}{4} H^{2} h^{2}-\frac{\lambda_{h}}{4} h^{4}-\frac{\lambda_{s}}{4} H^{4}-\frac{\eta_{\chi} \lambda_{h} v_{r} v_{\phi}}{m_{H}^{2}-m_{h}^{2}} H h^{3}+\frac{\eta_{\chi} \lambda_{s} v_{r} v_{\phi}}{m_{H}^{2}-m_{h}^{2}} H^{3} h \\
& -\lambda_{h} v_{r} h^{3}-\lambda_{s} v_{\phi} H^{3}-\frac{\eta_{\chi} v_{\phi}}{2}\left(\frac{6 \lambda_{h} v_{r}^{2}}{m_{H}^{2}-m_{h}^{2}}+1\right) H h^{2}+\frac{\eta_{\chi} v_{r}}{2}\left(\frac{6 \lambda_{s} v_{\phi}^{2}}{m_{H}^{2}-m_{h}^{2}}-1\right) H^{2} h
\end{aligned}
$$

Using (33) we can manipulate this expression to write the scalar potential as

$$
\begin{aligned}
\mathcal{V} & \approx \frac{1}{2} m_{H}^{2} H^{2}+\frac{1}{2} m_{h}^{2} h^{2} \\
& -\frac{\eta_{\chi}}{4} H^{2} h^{2}-\frac{\lambda_{h}}{4} h^{4}-\frac{\lambda_{s}}{4} H^{4}-\frac{\eta_{\chi} \lambda_{h} v_{r} v_{\phi}}{m_{H}^{2}-m_{h}^{2}} H h^{3}+\frac{\eta_{\chi} \lambda_{s} v_{r} v_{\phi}}{m_{H}^{2}-m_{h}^{2}} H^{3} h \\
& -\frac{m_{h}^{2}}{2 v_{r}} h^{3}-\frac{m_{h}^{2}}{2 v_{\phi}} H^{3}-\frac{\eta_{\chi} v_{\phi}}{2}\left(\frac{m_{H}^{2}+2 m_{h}^{2}}{m_{H}^{2}-m_{h}^{2}}\right) H h^{2}+\frac{\eta_{\chi} v_{r}}{2}\left(\frac{2 m_{H}^{2}+m_{h}^{2}}{m_{H}^{2}-m_{h}^{2}}\right) H^{2} h .
\end{aligned}
$$

Under the approximations taken previously, $m_{H} \gg m_{h}$ and $m_{H}^{2} \gg 2 \eta_{\chi} v_{r} v_{\phi}$, the relevant $H h h$ interaction term results in

$$
-\frac{\eta_{\chi} v_{\phi}}{2}\left(\frac{m_{H}^{2}+2 m_{h}^{2}}{m_{H}^{2}-m_{h}^{2}}\right) H h^{2} .
$$


The differential decay probability per unit time is given by

$$
d \Gamma_{H \rightarrow 2 h}=\frac{1}{2 m_{H}}\left(\frac{\eta_{\chi} v_{\phi}}{m_{H}^{2}-m_{h}^{2}}\right)^{2}\left(m_{H}^{2}+2 m_{h}^{2}\right)^{2} \frac{1}{2 !} \frac{k^{2} d k d \Omega}{(2 \pi)^{2} 4 E_{k}} \frac{1}{2 k} \delta\left(m_{h}-2 \sqrt{k^{2}+m_{H}^{2}}\right) .
$$

The partial $H \rightarrow 2 h$ decay width can now be expressed as

$$
\Gamma_{H \rightarrow 2 h}=\frac{1}{32 \pi m_{H}^{2}}\left(\frac{\eta_{\chi} v_{\phi}}{m_{H}^{2}-m_{h}^{2}}\right)^{2}\left(m_{H}^{2}+2 m_{h}^{2}\right)^{2} \sqrt{m_{H}^{2}-4 m_{h}^{2}} .
$$

In the limit $m_{H} \gg m_{h}$ we obtain

$$
\Gamma_{H \rightarrow 2 h}=\frac{1}{32 \pi}\left(\frac{\eta_{\chi} v_{\phi}}{m_{H}^{2}-m_{h}^{2}}\right)^{2} m_{H}^{3} .
$$

\section{C. $\Gamma_{H \rightarrow 2 w}$}

For $m_{w}<m_{H} / 2$, the $r-\phi$ mixing allows the Higgs boson to decay into pairs of the lightest $W$-WIMP. We obtain the invariant amplitude for this process (a description of Feynman rules for Majorana fermions can be found in Ref. [55]),

$$
i \mathcal{M}=i f \sin \chi \bar{u}(p) v\left(p^{\prime}\right)
$$

where $u(p)$ and $v(p)$ are Dirac spinors. The spin average rate is given by

$$
\sum_{s}|\mathcal{M}|^{2}=4 f^{2} \sin ^{2} \chi\left(p \cdot p^{\prime}-m_{w}^{2}\right)
$$

The partial $H$-decay rate into $2 w$ is

$$
\begin{aligned}
d \Gamma_{H \rightarrow 2 w} & =\frac{|\mathcal{M}|^{2}}{2 m_{H}} \frac{d^{3} p^{\prime}}{(2 \pi)^{3} 2 E_{p^{\prime}}} \frac{d^{3} p}{(2 \pi)^{3} 2 E_{p}}(2 \pi)^{4} \delta^{(3)}\left(\mathbf{p}^{\prime}+\mathbf{p}\right) \delta\left(m_{H}-p^{\prime}-p\right) \\
& =\frac{1}{2 !} \frac{d \Omega}{64 \pi^{2} m_{H}^{2}} \sqrt{m_{H}^{2}-4 m_{w}^{2}}|\mathcal{M}|_{\mathbf{p}^{\prime}=-\mathbf{p}, p=\sqrt{\left(m_{H} / 2\right)^{2}-m_{w}^{2}}}^{2}
\end{aligned}
$$

and so the partial width for this decay is given by

$$
\Gamma_{H \rightarrow 2 w}=\frac{2\left(m_{H}^{2}-4 m_{w}^{2}\right)}{32 \pi m_{H}^{2}}\left(\frac{f \eta_{\chi} v_{r} v_{\phi}}{m_{H}^{2}-m_{h}^{2}}\right)^{2} \sqrt{m_{H}^{2}-4 m_{w}^{2}} .
$$

For $m_{H} \gg 2 m_{w}$, (53) becomes

$$
\Gamma_{H \rightarrow 2 w}=\frac{1}{16 \pi}\left(\frac{f \eta_{\chi} v_{r} v_{\phi}}{m_{H}^{2}-m_{h}^{2}}\right)^{2} \sqrt{m_{H}^{2}-4 m_{w}^{2}}
$$




\section{D. $\Gamma_{H \rightarrow \text { hidden }}$}

All in all, the decay width of the Higgs into the hidden sector is given by

$$
\Gamma_{H \rightarrow \text { hidden }}=\frac{1}{16 \pi}\left(\frac{\eta_{\chi} v_{\phi}}{m_{H}^{2}-m_{h}^{2}}\right)^{2} m_{H}^{3}+\frac{1}{16 \pi}\left(\frac{f \eta_{\chi} v_{r} v_{\phi}}{m_{H}^{2}-m_{h}^{2}}\right)^{2} \sqrt{m_{H}^{2}-4 m_{w}^{2}} .
$$

Assuming $m_{H} \gg m_{h}$, this decay width is

$$
\Gamma_{H \rightarrow \text { hidden }}=\frac{\eta_{\chi}^{2} v_{\phi}^{2}}{16 \pi m_{H}}+\frac{\eta_{\chi}^{2} \Delta m^{2} v_{\phi}^{2}}{64 \pi m_{H}^{3}} .
$$

Comparing (34) and (56), we obtain

$$
|\Delta m| \lesssim 2 m_{H} \sqrt{\frac{8.3 \times 10^{-5}}{\eta_{\chi}^{2}}-1}
$$

which is satisfied if $\left|\eta_{\chi}\right|<0.009$.

\section{CONSTRAINTS FROM DIRECT DETECTION EXPERIMENTS}

Direct detection experiments attempt to observe the recoil from the elastic scattering of dark matter particles interacting with nuclei in the detector. Since the late 1990's the DAMA/NaI Collaboration [56] has been claiming to observe the expected annual modulation of the dark matter induced nuclear recoil rate due to the rotation of the Earth around the Sun [57, 58]. The upgraded DAMA/LIBRA detector confirmed [59] the earlier result adding many more statistics, and it has reached a significance of $8.9 \sigma$ for the cumulative exposure [23]. In 2010, the CoGeNT Collaboration reported an irreducible excess in the counting rate [24], which may also be ascribed to a dark matter signal. One year later, the same collaboration reported further data analyses showing that the time series of their rate is actually compatible with an annual modulation effect [25]. In CoGeNT data the evidence for the annual modulation is at the $2.8 \sigma$ level. In the summer of 2011, the CRESST Collaboration also reported an excess of low energy events that are not consistent with known backgrounds [26]. In particular, 67 counts were found in the dark matter acceptance region and the estimated background from leakage of $e / \gamma$ events, neutrons, $\alpha$ particles, and recoiling nuclei in $\alpha$ decays is not sufficient to account for all the observed events. The CRESST Collaboration rejected the background-only hypothesis at more than $4 \sigma$. Of particular interest here, the DAMA (after including the effect of channeling in the NaI crystal scintillators [60]) and CoGeNT results appear to be compatible with a relatively light dark matter particle, in the few $\mathrm{GeV}$ to tens of $\mathrm{GeV}$ mass range, with a scattering cross section against nucleons of about $7 \times 10^{-41} \mathrm{~cm}^{2}$ [28-31]. The central value favored by CRESST data points to somewhat larger dark matter masses, but it is still compatible at the $1 \sigma$ level with the range determined by the other two experiments.

Very recently, CDMS II Collaboration reported three candidate events with an expected background of 0.7 events [27]. If interpreted as a signal of elastically scattering dark matter, the central value of the likelihood analysis of the measured recoil energies favors a mass of $8.6 \mathrm{GeV}$ and a scattering cross section on nucleons of

$$
\sigma_{w N}^{m_{w} \approx 10 \mathrm{GeV}} \approx 1.9 \times 10^{-41} \mathrm{~cm}^{2} .
$$


The $68 \%$ confidence band is somewhat large and overlaps with previous signal claims.

Alongside these "signals" stands the series of null results from the XENON-100 [34] and XENON-10 [61] experiments, which at present have the world's strongest exclusion limit. Some authors have pointed out that uncertainties in the response of liquid xenon to low energy nuclear recoil may be significant, particularly in the mass region of interest [62, 63]. In light of these suspicions, a recent reanalysis of XENON data suggests candidates in fact may have been observed [35]. The data favor a mass of $12 \mathrm{GeV}$, though the $90 \%$ error contours extend from 7 to $30 \mathrm{GeV}$ with the cross section varying between $6 \times 10^{-41} \mathrm{~cm}^{2}$ and $4 \times 10^{-45} \mathrm{~cm}^{2}$. Taken together, these different arguments suggest that the existing data set is not inconsistent with a dark matter candidate of about $10 \mathrm{GeV}$.

The $w N$ cross section for elastic scattering is given by

$$
\sigma_{w N}=\frac{4}{\pi} \frac{m_{w}^{2} m_{N}^{2}}{\left(m_{w}+m_{N}\right)^{2}} \frac{f_{p}^{2}+f_{n}^{2}}{2},
$$

where $N \equiv \frac{1}{2}(n+p)$ is an isoscalar nucleon, in the renormalization-group-improved parton model $[64,65]$. The effective couplings to protons $f_{p}$ and neutrons $f_{n}$ are given by

$$
f_{p, n}=\sum_{q=u, d, s} \frac{G_{q}}{\sqrt{2}} f_{T q}^{(p, n)} \frac{m_{p, n}}{m_{q}}+\frac{2}{27} f_{T G}^{(p, n)} \sum_{q=c, b, t} \frac{G_{q}}{\sqrt{2}} \frac{m_{p, n}}{m_{q}},
$$

where $G_{q}$ is the $W$-WIMP's effective Fermi coupling for a given quark species,

$$
\mathscr{L}=\frac{G_{q}}{\sqrt{2}} \bar{\psi}_{-} \psi_{-} \bar{\psi}_{q} \psi_{q}
$$

with $\psi_{q}$ the SM quark field of flavor $q$. The first term in (60) reflects scattering with light quarks, whereas the second term accounts for interaction with gluons through a heavy quark loop. The terms $f_{T q}^{(p, n)}$ are proportional to the matrix element, $\langle\bar{q} q\rangle$, of quarks in a nucleon, and are given by

$$
\begin{array}{cll}
f_{T u}^{p}=0.020 \pm 0.004, & f_{T d}^{p}=0.026 \pm 0.005, & f_{T s}^{p}=0.118 \pm 0.062 \\
f_{T u}^{n}=0.014 \pm 0.003, & f_{T d}^{n}=0.036 \pm 0.008, & f_{T s}^{n}=0.118 \pm 0.062
\end{array}
$$

We also have $f_{T G}^{(p, n)}=1-\sum_{u, d, s} f_{T q}^{(p, n)}$, which is $f_{T G}^{p} \approx 0.84$ and $f_{T G}^{n} \approx 0.83$ [64].

To establish the value of $G_{q} / m_{q}$ we look back at (32) along with the SM Yukawa interaction term, which involves the mixing of both scalar fields, $H$ and $h$. For interactions of $W$-WIMPs with SM quarks, the relevant terms are

$$
\mathscr{L}=\frac{m_{q} \cos \chi}{v_{\phi}} H \bar{\psi}_{q} \psi_{q}-\frac{m_{q} \sin \chi}{v_{\phi}} h \bar{\psi}_{q} \psi_{q}+\cdots+\frac{f \sin \chi}{2} H \bar{\psi}_{-} \psi_{-}+\frac{f \cos \chi}{2} h \bar{\psi}_{-} \psi_{-} .
$$

The scattering of a $w$ particle off a quark then gives

$$
\begin{aligned}
\mathcal{M} & =i \frac{f m_{q} \sin \chi \cos \chi}{v_{\phi}} \bar{u}_{q}\left(p^{\prime}\right) u_{q}(p)\left(\frac{1}{t-m_{H}^{2}}-\frac{1}{t-m_{h}^{2}}\right) \bar{u}\left(k^{\prime}\right) u(k) \\
& \approx i \frac{f m_{q} \eta_{\chi} v_{r}}{m_{H}^{2} m_{h}^{2}} \bar{u}_{q}\left(p^{\prime}\right) u_{q}(p) \bar{u}\left(k^{\prime}\right) u(k) \\
& \approx i \frac{m_{q} \eta_{\chi} \Delta m}{2 m_{H}^{2} m_{h}^{2}} \bar{u}_{q}\left(p^{\prime}\right) u_{q}(p) \bar{u}\left(k^{\prime}\right) u(k)
\end{aligned}
$$


This leads to the identification of the effective coupling

$$
\frac{2 G_{q}}{\sqrt{2}}=\frac{m_{q} \eta_{\chi} \Delta m}{2 m_{H}^{2} m_{h}^{2}} \Rightarrow \frac{G_{q}}{m_{q}}=\frac{\eta_{\chi} \Delta m}{2 \sqrt{2} m_{H}^{2} m_{h}^{2}} .
$$

The insertion of (65) and (60) into (59) yields

$$
\sigma_{w N} \approx 3 \times 10^{-7}\left[\frac{226.27 \eta_{\chi} \Delta m \mathrm{GeV}}{m_{h}^{2}}\right]^{2} \mathrm{pb} .
$$

Combining (66) with the signals/bounds on elastic scattering of dark matter particles on nucleons we obtain a constraining relation for $\eta_{\chi} \Delta m$. For $m_{w}=10 \mathrm{GeV}$, we use the cross section reported by the CDMS Collaboration (58) to obtain

$$
\eta_{\chi} \Delta m=\frac{3.5 \times 10^{-2}}{\mathrm{GeV}} m_{h}^{2}
$$

For $m_{w}=50 \mathrm{GeV}$, we adopt the $90 \%$ C.L. upper limit reported by the XENON-100 Collaboration [34] to obtain

$$
\eta_{\chi} \Delta m<\frac{3.6 \times 10^{-4}}{\mathrm{GeV}} m_{h}^{2}
$$

\section{CONSTRAINTS FROM COSMOLOGICAL OBSERVATIONS}

The concordance model of cosmology predicts the evolution of a spatially flat expanding Universe filled with dark energy, dark matter, baryons, photons, and three flavors of lefthanded (i.e. one helicity state $\nu_{L}$ ) neutrinos (along with their right-handed antineutrinos $\bar{\nu}_{R}$ ). The universal expansion rate, quantified by the Hubble parameter $H$, is determined by the total energy density $\rho$,

$$
H^{2} \equiv \frac{\dot{a}}{a}=\frac{8 \pi G}{3} \rho,
$$

where $a$ is the expansion scale factor and $G$ is the gravitational constant. In the relatively late, early Universe, the energy density is dominated by radiation, that is by the contributions from massless and/or extremely relativistic particles (i.e. $\rho \approx \rho_{\mathrm{R}}$ ).

The earliest observationally verified landmarks - big bang nucleosynthesis (BBN) and the cosmic microwave background (CMB) decoupling epoch - have become the de facto worldwide standard for probing theoretical scenarios beyond the SM containing new light species. It is advantageous to normalize the extra contribution to the SM energy density to that of an "equivalent" neutrino species. The number of equivalent light neutrino species,

$$
N_{\mathrm{eff}}=\frac{\rho_{\mathrm{R}}-\rho_{\gamma}}{\rho_{\nu_{L}}}
$$

quantifies the total "dark" relativistic energy density (including the three left-handed SM neutrinos) in units of the density of a single Weyl neutrino

$$
\rho_{\nu_{L}}=\frac{7 \pi^{2}}{120}\left(\frac{4}{11}\right)^{4 / 3} T_{\gamma}^{4}
$$




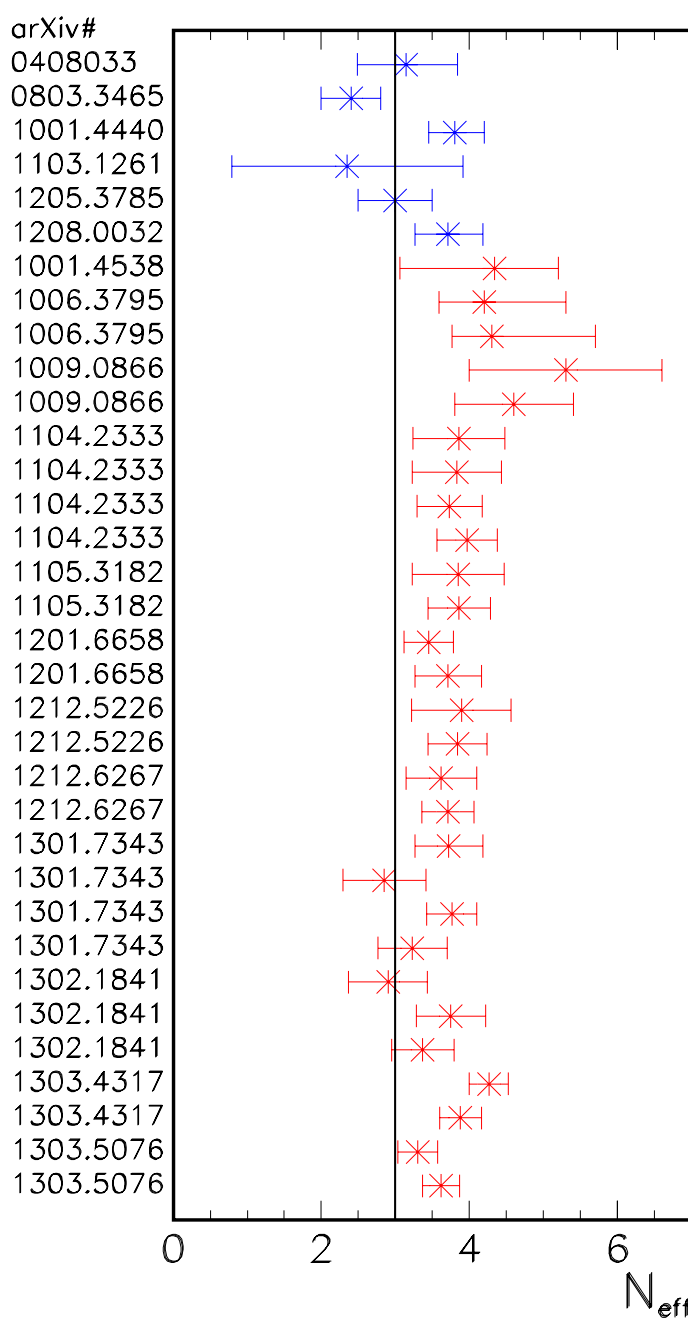

$\mathrm{BBN}$ and $\mathrm{CMB}$ observations

$\mathrm{D} / \mathrm{H}+{ }^{4} \mathrm{He}$

$\mathrm{D} / \mathrm{H}+{ }^{4} \mathrm{He}$

${ }^{4} \mathrm{He}$

D $/ \mathrm{H}+{ }^{4} \mathrm{He}$

$\mathrm{D} / \mathrm{H}$

D $/ \mathrm{H}+{ }^{4} \mathrm{He}$

WMAP $7+\mathrm{H}_{0}+\mathrm{BAO}$

WMAP7+CBI+VSA+BOOMERANG +ACBAR+ $\mathrm{H}_{0}+\mathrm{SN}+\mathrm{BAO}$

WMAP7+CBI+VSA+BOOMERANG +ACBAR+ $\mathrm{H}_{0}+\mathrm{SN}+\mathrm{SDSS}$

WMAP7 +ACT

WMAP $7+\mathrm{ACT}+\mathrm{H}_{0}+\mathrm{BAO}$

SPT + WMAP7

SPT + WMAP7 +BAO

$\mathrm{SPT}+\mathrm{WMAP} 7+\mathrm{H}_{0}$

$\mathrm{SPT}+\mathrm{WMAP} 7+\mathrm{H}_{0}+\mathrm{BAO}$

SPT + WMAP7

$\mathrm{SPT}+\mathrm{WMAP} 7+\mathrm{H}_{0}+\mathrm{BAO}$

SPT +WMAP $7+\mathrm{H}(z)$

WMAP7 $+A C T+H(z)$

$\mathrm{SPT}+$ WMAP $+\mathrm{ACT}$

$\mathrm{SPT}+\mathrm{WMAP9}+\mathrm{ACT}+\mathrm{H}_{0}+\mathrm{BAO}$

SPT + WMAP7

$\mathrm{SPT}+\mathrm{WMAP} 7+\mathrm{H}_{0}+\mathrm{BAO}$

SPT + WMAP9

WMAP9 + ACT

$\mathrm{SPT}+\mathrm{WMAPO}+\mathrm{H}_{0}+\mathrm{BAO}$

WMAP9 $+\mathrm{ACT}+\mathrm{H}_{0}+\mathrm{BAO}$

WMAP9 +ACT

SPT + WMAP9

SPT +WMAP9 +ACT

SPT +WMAP9 $+\mathrm{H}_{0}+\mathrm{BAO}$

WMAP $9+\mathrm{ACT}+\mathrm{H}_{0}+\mathrm{BAO}$

$\mathrm{PLANCK}+\mathrm{SPT}+$ WMAP + ACT + BAO

$\mathrm{PLANCK}+\mathrm{SPT}+\mathrm{WMAP}+\mathrm{ACT}+\mathrm{H}_{0}$

FIG. 1: A selection of the most recent cosmological $N_{\text {eff }}$ measurements and the $1 \sigma$ confidence intervals from various combinations of models and data sets. BBN findings [67-72] are shown in blue and those from the CMB epoch [73-84] in red.

where $\rho_{\gamma}$ is the energy density of photons (which by today have redshifted to become the $\mathrm{CMB}$ photons at a temperature of about $\left.T_{\gamma}^{\text {today }} \simeq 2.7 \mathrm{~K}\right)[66]$.

A selection of the most recent measurements of $N_{\text {eff }}$ together with the $1 \sigma$ confidence intervals from various combinations of models and data sets are shown in Fig. 1. Altogether, the data hint at the presence of an excess $\Delta N$ above SM expectation of $N_{\text {eff }}=3.046$ [85]. Arguably, one of the most intriguing results of the Planck spacecraft is that the best-fit Hubble constant has the value $h=0.674 \pm 0.012$ [84]. ${ }^{2}$ This result deviates by more than $2 \sigma$ from the value obtained with the Hubble Space Telescope, $h=0.738 \pm 0.024$ [86]. The impact of the new $h$ determination is particularly complex in the investigation of $N_{\text {eff }}$. Combining CMB observations with data from baryon acoustic oscillations (BAO) [87], the

\footnotetext{
${ }^{2}$ We adopt the usual convention of writing the Hubble constant at the present day as $H_{0}=$ $100 h \mathrm{~km} \mathrm{~s}^{-1} \mathrm{Mpc}^{-1}$. There is some notational overlap with the differences of the massive states of the $r$ and $\phi$ fields; however, the context should make it clear what we are using.
} 
Planck Collaboration reported $N_{\text {eff }}=3.30 \pm 0.27$. Adding the $H_{0}$ measurement to the CMB data relieves the tension between the $\mathrm{CMB}$ data and $H_{0}$ at the expense of new neutrinolike physics (at around the $2.3 \sigma$ level), that is $N_{\text {eff }}=3.62 \pm 0.25$.

As noted in [41], the Goldstone boson $\alpha$ is a natural candidate for an imposter equivalent neutrino. The contribution of $\alpha$ to $N_{\text {eff }}$ is $\Delta N=\rho_{\alpha} / \rho_{\nu_{L}}$. Thus, taking into account the isentropic heating of the rest of the plasma between $T_{\alpha}^{\text {dec }}$ and $T_{\nu_{L}}^{\text {dec }}$ decoupling temperatures we obtain

$$
\Delta N=\frac{4}{7}\left(\frac{g\left(T_{\nu_{L} \mathrm{dec}}^{\mathrm{dec}}\right)}{g\left(T_{\alpha}^{\mathrm{dec}}\right)}\right)^{4 / 3}
$$

where $g(T)$ is the effective number of interacting (thermally coupled) relativistic degrees of freedom at temperature $T$; for example, $g\left(T_{\nu_{L}}^{\mathrm{dec}}\right)=43 / 4$ [88]. ${ }^{3}$ For the particle content of the SM, there is a maximum of $g\left(T_{\alpha}^{\text {dec }}\right)=427 / 4$ (with $T_{\alpha}^{\text {dec }}>m_{t}$ ), which corresponds to a minimum value of $\Delta N_{\nu}=0.027$.

We now turn to calculating the interaction rate for Goldstone bosons,

$$
\Gamma(T)=\sum_{\text {fermions }} n_{\mathrm{f}}(T)\langle\sigma v\rangle
$$

where

$$
n_{\mathrm{f}}(T)=\frac{g_{\mathrm{f}}}{2 \pi^{2}} \int_{0}^{\infty} \frac{k^{2}}{e^{\beta \sqrt{k^{2}+m_{\mathrm{f}}^{2}}}+1} d k
$$

is the number density of an interacting fermion of type $\mathrm{f}$ (with mass $m_{\mathrm{f}}$ ), $\beta=\left(k_{B} T\right)^{-1}, g_{\mathrm{f}}$ is the number of chiral states, and we average the cross section over the statistical distribution for a given temperature. For $T \gg m_{\mathrm{f}}$, we obtain

$$
n_{\mathrm{f}}(T) \approx g_{\mathrm{f}} \frac{3 \zeta(3)}{4 \pi^{2}}\left(\frac{k_{B} T}{\hbar c}\right)^{3} .
$$

This results in a simplification of (73)

$$
\Gamma(T) \approx \frac{3 \zeta(3)}{4 \pi^{2}}\left(\frac{k_{B} T}{\hbar c}\right)^{3} \sum_{\text {fermions }} g_{\mathrm{f}}\langle\sigma v\rangle .
$$

Since the Goldstone boson only interacts with the SM fields via the Higgs, we can have scatterings of the type $\alpha \psi \rightarrow \alpha \psi$, with $\psi$ a generic SM fermion. The $\alpha$ scattering off fermions is described by SM Yukawa interaction terms that can be written as

$$
\begin{aligned}
Y_{\mathrm{f}} \phi \bar{\psi} \psi & \rightarrow Y_{\mathrm{f}} v_{\phi} \bar{\psi} \psi+Y_{\mathrm{f}} \phi^{\prime} \bar{\psi} \psi, \\
& =m_{\mathrm{f}} \bar{\psi} \psi+\frac{m_{\mathrm{f}}}{v_{\phi}} H \bar{\psi} \psi \cos \chi-\frac{m_{\mathrm{f}}}{v_{\phi}} h \bar{\psi} \psi \sin \chi
\end{aligned}
$$

where $Y_{\mathrm{f}}$ is the Yukawa coupling of the fermion in question.

\footnotetext{
${ }^{3}$ If relativistic particles are present that have decoupled from the photons, it is necessary to distinguish between two kinds of $g: g_{\rho}$, which is associated with the total energy density, and $g_{s}$, which is associated with the total entropy density. For our calculations we use $g=g_{\rho}=g_{s}$.
} 
We proceed to calculate the scattering cross section. The invariant amplitude follows from the Feynman rules

$$
i \mathcal{M}=\frac{2 m_{f} \sin \chi \cos \chi}{v_{r} v_{\phi}}\left(k \cdot k^{\prime}\right) \frac{i}{t-m_{H}^{2}} \bar{u}\left(p^{\prime}\right) u(p)-\frac{2 m_{f} \cos \chi \sin \chi}{v_{r} v_{\phi}}\left(k \cdot k^{\prime}\right) \frac{i}{t-m_{h}^{2}} \bar{u}\left(p^{\prime}\right) u(p) .
$$

The momenta of incoming and outgoing (outgoing primed) particles are defined by

$$
\begin{aligned}
p^{\mu} & =(p, p \sin \varphi, 0,-p \cos \varphi) \\
k^{\mu} & =(k, 0,0, k) \\
k^{\prime \mu} & =\left(k^{\prime}, k^{\prime} \sin \vartheta, 0, k^{\prime} \cos \vartheta\right) \\
p^{\prime \mu} & =\left(p^{\prime},-p^{\prime} \sin \vartheta^{\prime}, 0,-p^{\prime} \cos \vartheta^{\prime}\right)
\end{aligned}
$$

with $t=p^{\prime}-p$. To obtain the (unpolarized) cross section, we have to take the square of the modulus of $\mathcal{M}$ and then carry out the spin and color (if appropriate) sums

$$
\frac{1}{2} \sum_{\text {spins, colors }}|\mathcal{M}|^{2}=8 N_{c}\left(\frac{m_{f} \sin \chi \cos \chi}{v_{r} v_{\phi}}\right)^{2}\left(\frac{m_{H}^{2}-m_{h}^{2}}{\left(t-m_{H}^{2}\right)\left(t-m_{h}^{2}\right)}\right)^{2}\left(k \cdot k^{\prime}\right)^{2}\left(p \cdot p^{\prime}+m_{f}^{2}\right),
$$

where $N_{c}=3$ for quarks and $N_{c}=1$ for leptons. The cross section in the center-of-mass (c.m.) frame in the highly relativistic approximation is given by

$$
\frac{d \sigma}{d \Omega} \approx \frac{N_{c}}{8 \pi^{2} s}\left(\frac{m_{f} \eta_{\chi}}{\left(t-m_{H}^{2}\right)\left(t-m_{h}^{2}\right)}\right)^{2}\left(k \cdot k^{\prime}\right)^{2}\left(p \cdot p^{\prime}\right)
$$

where $s=(k+p)^{2} \approx 4 k^{2}$ and finally $\eta_{\chi} \ll 1$. To make progress on this problem we take the effective coupling form

$$
\sigma(s) \approx \frac{N_{c}}{64 \pi}\left(\frac{m_{f} \eta_{\chi}}{m_{H}^{2} m_{h}^{2}}\right)^{2} s^{2} .
$$

Nonequilibrium thermal physics tells us that the way to do thermal averaging within Boltzmann's approximation is

$$
\begin{aligned}
\langle\sigma v\rangle & =\int d \Pi_{p^{\prime}} d \Pi_{k^{\prime}} d \Pi_{k} d \Pi_{p}\left|\mathcal{M}\left(k+p \rightarrow k^{\prime}+p^{\prime}\right)\right|^{2} f_{\mathrm{f}}(p, T) f_{\alpha}(k, T) \\
& \times(2 \pi)^{4} \delta^{(4)}\left(p+k-p^{\prime}-k^{\prime}\right)
\end{aligned}
$$

with $d \Pi_{p}=d^{3} p^{\prime} /\left[(2 \pi)^{3} 2 E_{p^{\prime}}\right]$ and likewise for the other parameters. Here, $f_{\mathrm{f}}$ and $f_{\alpha}$ are Fermi and Bose equilibrium normalized distributions, corresponding to the $\mathrm{f}$ fermion and $\alpha$ boson, respectively. The expression from nonequilibrium thermal physics [Eq. (83)] is approximated by

$$
\langle\sigma v\rangle \approx \int \frac{d^{3} k}{(2 \pi)^{3}} \frac{d^{3} p}{(2 \pi)^{3}} f_{\mathrm{f}}(p, T) f_{\alpha}(k, T) v_{M} \sigma(s),
$$

where $v_{M} \approx k \cdot p /(p k)=2(1+\cos \varphi)$ is the Möller velocity in the ultrarelativistic limit $[89,90]$ and $s=2 k p(1+\cos \varphi)$ is the c.m. energy of two interacting particles with initial momenta 
not necessarily collinear. The velocity average cross section then is found to be

$$
\begin{aligned}
\langle\sigma v\rangle & \approx \frac{1}{8 \pi^{4}} \int_{0}^{\infty} p^{2} d p \int_{0}^{\infty} k^{2} d k \int_{0}^{\pi} \sin \varphi d \varphi f_{\mathrm{f}}(p, T) f_{\alpha}(k, T) \\
& \times 2(1+\cos \varphi) \sigma_{\mathrm{c} . \mathrm{m} .}[2 k p(1+\cos \varphi)], \\
& =N_{c} \frac{15 \zeta^{2}(5)}{\pi \zeta^{2}(3)}\left(\frac{m_{f} \eta_{\chi}}{m_{H}^{2} m_{h}^{2}}\right)^{2}\left(k_{B} T\right)^{4}, \\
& \approx 3.55 N_{c}\left(\frac{m_{f} \eta_{\chi}}{m_{H}^{2} m_{h}^{2}}\right)^{2}\left(k_{B} T\right)^{4} .
\end{aligned}
$$

Putting this all together, we obtain

$$
\Gamma(T) \approx 0.32\left(\frac{\eta_{\chi}}{m_{H}^{2} m_{h}^{2}}\right)^{2}\left(k_{B} T\right)^{7} \sum_{\text {fermions }} g_{\mathrm{f}} N_{\mathrm{c}} m_{\mathrm{f}}^{2} .
$$

Now, since we can approximate the energy density (at high temperatures) by including only particles species $i$ with $T \gg m_{i}$, it follows that

$$
\rho_{\mathrm{R}}=\left(\sum_{\text {bosons }} g_{\mathrm{b}}+\frac{7}{8} \sum_{\text {fermions }} g_{\mathrm{f}}\right) \frac{\pi^{2}}{30}\left(k_{B} T\right)^{4}=\frac{\pi^{2}}{30} g(T)\left(k_{B} T\right)^{4}
$$

and therefore the Hubble parameter (69) becomes

$$
H(T) \simeq \frac{1.66}{M_{\mathrm{Pl}}} \sqrt{g(T)}\left(k_{B} T\right)^{2}
$$

where $g_{\mathrm{b}(\mathrm{f})}$ is the number of degrees of freedom of each boson (fermion) and the sum runs over all boson and fermion states with $T \gg m_{i}$. The factor of $7 / 8$ is due to the difference between the Fermi and Bose integrals.

The Goldstone boson decouples from the plasma when its mean free path becomes greater than the Hubble radius at that time

$$
\Gamma\left(T_{\alpha}^{\mathrm{dec}}\right)=H\left(T_{\alpha}^{\mathrm{dec}}\right) .
$$

The most interesting thermodynamics originates if $\alpha$ goes out of thermal equilibrium while $T$ is still above the mass of the muons but below the mass of all other particles of the SM, a time when neutrinos are still in thermal equilibrium. For instance, with $\eta_{\chi}=0.005$ and $m_{h} \approx 500 \mathrm{MeV}$ we obtain [41]

$$
\Delta N=(4 / 7)(43 / 57)^{4 / 3}=0.39 .
$$

This corresponds to a number of equivalent light neutrino species that is consistent at the $1 \sigma$ level with both the estimate of $N_{\text {eff }}$ using Planck + BAO data as well as the estimate using Planck $+H_{0}$ data.

However, of particular interest here is the case where the mass of the Goldstone boson companion field is $m_{h} \approx 98 \mathrm{GeV}$ and $\eta_{\chi}=0.0003$. For such set of parameters, $\alpha$ decouples when

$$
0.32\left(\frac{\eta_{\chi}}{m_{H}^{2} m_{h}^{2}}\right)^{2}\left(k_{B} T\right)^{7} 12 m_{b}^{2}=\frac{1.66}{M_{\mathrm{Pl}}} \sqrt{86.25}\left(k_{B} T\right)^{2},
$$


where we have approximated $\sum_{\text {fermions }} N_{c} g_{\mathrm{f}} m_{\mathrm{f}}^{2} \approx 12 m_{b}^{2}$. This gives $T \approx 5 \mathrm{GeV}$, and so the $\alpha$ contribution to $N_{\text {eff }}$ is found to be

$$
\Delta N \approx 0.036
$$

The corresponding value of $N_{\text {eff }}$ is within the $1 \sigma$ interval of the value reported by the Planck Collaboration using Planck + BAO data but far out from the value derived using Planck + $H_{0}$ data. Should future data point toward the Planck $+H_{0}$ value, one should find a different origin to explain the extra relativistic degrees of freedom (if $m_{h} \approx 98 \mathrm{GeV}$ ). One interesting possibility is to include the right-handed partners of the three left-handed, SM neutrinos. It was shown elsewhere [91-95] that milliweak interactions of these Dirac states (through their coupling to a TeV-scale $Z^{\prime}$ gauge boson) may allow the $\nu_{R}$ 's to decouple during the course of the quark-hadron crossover transition, just so that they are partially reheated compared to the $\nu_{L}$ 's. Remarkably, the required mass for the $Z^{\prime}$ gauge boson is within the range of discovery of LHC.

\section{FITTING FERMI DATA AND THE OBSERVED DARK MATTER DENSITY}

Next, in line with our stated plan, we use Fermi data and the observed relic density to determine the free parameters of the model. To this end we first calculate the annihilation rate into SM fermions and Goldstone bosons.

\section{A. W-WIMP Annihilation into SM Fermions}

The $W$-WIMP can annihilate into SM fermions via $\bar{\psi}_{-} \psi_{-} \rightarrow \phi^{*} / r^{*} \rightarrow \bar{\psi} \psi$, with an $s$-channel Higgs or $h$ mediator. The matrix element of this process is given by

$$
i \mathcal{M}=i f \sin \chi \cos \chi \bar{v}\left(p^{\prime}\right) u(p)\left(\frac{i}{s-m_{H}^{2}}-\frac{i}{s-m_{h}^{2}}\right) \frac{i m_{\mathrm{f}}}{v_{\phi}} \bar{u}\left(k^{\prime}\right) v(k) .
$$

The minus sign in the second propagator is necessary because the $r$ couples with a negative sign to fermions compared to the Higgs; see (77). The spin-averaged invariant amplitude reads

$$
\frac{1}{4} \sum|\mathcal{M}|^{2}=N_{c}\left(\frac{f m_{\mathrm{f}} \sin \chi \cos \chi}{v_{\phi}}\right)^{2} \frac{4\left(m_{h}^{2}-m_{H}^{2}\right)^{2}\left(p \cdot p^{\prime}-m_{w}^{2}\right)\left(k \cdot k^{\prime}-m_{\mathrm{f}}^{2}\right)}{\left(s-m_{h}^{2}\right)^{2}\left(s-m_{H}^{2}\right)^{2}} .
$$

Now, let us calculate the cross section for $\mathrm{f} \bar{f}$-pair production

$$
\begin{aligned}
d \sigma & =\frac{1}{8 E_{p}|p|}|\mathcal{M}|^{2} \frac{d^{3} k}{(2 \pi)^{3} 2 E_{k}} \frac{d^{3} k^{\prime}}{(2 \pi)^{3} 2 E_{k^{\prime}}}(2 \pi)^{4} \delta^{(3)}\left(\mathbf{k}^{\prime}+\mathbf{k}\right) \\
& \times \delta\left(2 E_{p}-E_{k}-E_{k^{\prime}}\right)
\end{aligned}
$$


and so

$$
\begin{aligned}
\sigma & =\frac{|\mathcal{M}|^{2}}{64 \pi} \frac{\left|k^{\prime}\right|}{|p| E_{p}^{2}} \\
& =\frac{N_{c}}{16 \pi}\left(\frac{f m_{\mathrm{f}} \sin \chi \cos \chi}{v_{\phi}}\right)^{2} \frac{\left|k^{\prime}\right|}{|p|} \frac{\left(m_{h}^{2}-m_{H}^{2}\right)^{2}}{\left(s-m_{h}^{2}\right)^{2}\left(s-m_{H}^{2}\right)^{2}} \frac{\left(p \cdot p^{\prime}-m_{w}^{2}\right)\left(k \cdot k^{\prime}-m_{\mathrm{f}}^{2}\right)}{E_{p}^{2}} \\
& \approx \frac{N_{c}}{16 \pi}\left(\frac{\eta_{\chi} m_{\mathrm{f}} \Delta m}{2\left(s-m_{h}^{2}\right)\left(s-m_{H}^{2}\right)}\right)^{2} \sqrt{\frac{\left|s-4 m_{\mathrm{f}}^{2}\right|}{\left|s-4 m_{w}^{2}\right|}} \frac{\left(s-4 m_{w}^{2}\right)\left(s-4 m_{\mathrm{f}}^{2}\right)}{s} .
\end{aligned}
$$

In this case the out state does not consist of identical particles. For phenomenological purposes, the $h$ pole needs to be softened to a Breit-Wigner form by obtaining and utilizing the correct total widths $\Gamma_{h}$ of the resonance. This is accomplished by modification of the $s$-channel propagator for $h$ via

$$
\frac{i}{s-m_{h}^{2}} \rightarrow \frac{i}{s-m_{h}^{2}-i m_{h} \Gamma_{h}}
$$

After this is done, the contribution of the $f \bar{f}$ channel is as follows:

$$
\begin{aligned}
\sigma & =\frac{N_{c}}{16 \pi}\left(\frac{\eta_{\chi} m_{\mathrm{f}} \Delta m}{2\left(m_{H}^{2}-m_{h}^{2}\right)\left(s-m_{H}^{2}\right)}\right)^{2} \frac{\left(m_{H}^{2}-m_{h}^{2}\right)^{2}+m_{h}^{2} \Gamma_{h}^{2}}{\left(s-m_{h}^{2}\right)^{2}+m_{h}^{2} \Gamma_{h}^{2}} \sqrt{\frac{\left|s-4 m_{\mathrm{f}}^{2}\right|}{\left|s-4 m_{w}^{2}\right|}} \\
& \times \frac{\left(s-4 m_{w}^{2}\right)\left(s-4 m_{\mathrm{f}}^{2}\right)}{s}, \\
& \approx \frac{N_{c}}{16 \pi}\left(\frac{\eta_{\chi} m_{\mathrm{f}} \Delta m}{2\left(s-m_{H}^{2}\right)}\right)^{2} \frac{1}{\left(s-m_{h}^{2}\right)^{2}+m_{h}^{2} \Gamma_{h}^{2}} \sqrt{\frac{\left|s-4 m_{\mathrm{f}}^{2}\right|}{\left|s-4 m_{w}^{2}\right|}} \frac{\left(s-4 m_{w}^{2}\right)\left(s-4 m_{\mathrm{f}}^{2}\right)}{s} .
\end{aligned}
$$

For $\Delta m>m_{H} / 2$, the decay channels of the $h$ field are $h \rightarrow \mathrm{f} \bar{f}, h \rightarrow w \bar{w}$, and $h \rightarrow 2 \alpha^{\prime}$. The corresponding decay widths are given by

$$
\begin{aligned}
\Gamma_{h \rightarrow \mathrm{f} \overline{\mathrm{f}}} & =\sum_{\text {fermions }} \frac{N_{c}}{8 \pi m_{h}^{2}}\left(\frac{m_{\mathrm{f}} \sin \chi}{v_{\phi}}\right)^{2}\left(m_{h}^{2}-4 m_{\mathrm{f}}^{2}\right)^{3 / 2} \\
& \approx \sum_{\text {fermions }} \frac{N_{c}}{8 \pi m_{h}^{2}}\left(\frac{m_{\mathrm{f}} \eta_{\chi} v_{r}}{m_{H}^{2}-m_{h}^{2}}\right)^{2}\left(m_{h}^{2}-4 m_{\mathrm{f}}^{2}\right)^{3 / 2} \\
& \approx \sum_{\text {fermions }} \frac{N_{c}}{8 \pi m_{h}^{2} f^{2}}\left(\frac{m_{\mathrm{f}} \eta_{\chi} \Delta m}{2\left(m_{H}^{2}-m_{h}^{2}\right)}\right)^{2}\left(m_{h}^{2}-4 m_{\mathrm{f}}^{2}\right)^{3 / 2} \\
& \approx \frac{3}{8 \pi m_{h}^{2} f^{2}}\left(\frac{m_{\mathrm{b}} \eta_{\chi} \Delta m}{2\left(m_{H}^{2}-m_{h}^{2}\right)}\right)^{2}\left(m_{h}^{2}-4 m_{\mathrm{f}}^{2}\right)^{3 / 2}
\end{aligned}
$$

(in the last line we have taken $m_{b}<m_{w}<m_{t}$ ),

$$
\begin{aligned}
\Gamma_{h \rightarrow w \bar{w}} & =\frac{2\left(m_{h}^{2}-4 m_{w}^{2}\right) f^{2} \cos ^{2} \chi}{32 \pi m_{h}^{2}} \sqrt{m_{h}^{2}-4 m_{w}^{2}} \\
& \approx \frac{f^{2}}{16 \pi m_{h}^{2}}\left(m_{h}^{2}-4 m_{w}^{2}\right)^{3 / 2}
\end{aligned}
$$


(inclusion of this channel requires $2 m_{w}<m_{h}$ ), and

$$
\begin{aligned}
\Gamma_{h \rightarrow 2 \alpha^{\prime}} & =\frac{1}{32 \pi}\left(\frac{\cos \chi}{v_{r}}\right)^{2} m_{h}^{3} \\
& \approx \frac{f^{2}}{8 \pi \Delta m^{2}} m_{h}^{3}
\end{aligned}
$$

The dominant terms of the total decay width come from the hidden sector. Hence, in what follows we neglect terms accounting for $h$ decay into the visible sector and consider $m_{h}<2 m_{w}$ (so that the decay $h \rightarrow w \bar{w}$ is closed). Under these assumptions the decay width takes a particularly simple form

$$
\Gamma_{h}=\frac{f^{2}}{8 \pi \Delta m^{2}} m_{h}^{3} .
$$

Next, we compute the averaged cross section for thermal interactions. In the cosmic comoving frame (the frame where the gas is assumed to be at rest as a whole) we have

$$
\langle\sigma v\rangle=\frac{\int d^{3} p d^{3} p^{\prime} f_{w}(p, T) f_{w}\left(p^{\prime}, T\right) \sigma v_{M}}{\int d^{3} p d^{3} p^{\prime} f_{w}(p, T) f_{w}\left(p^{\prime}, T\right)},
$$

where $\boldsymbol{p}$ and $\boldsymbol{p}^{\prime}$ are the three-momenta of the colliding particles, whose equilibrium distribution function at temperature $T$ is Maxwell-Boltzmann,

$$
f_{w}(p, T) \approx e^{-\beta \sqrt{p^{2}+m_{w}^{2}}}
$$

with $p=|\boldsymbol{p}|$ and $p^{\prime}=\left|\boldsymbol{p}^{\prime}\right|$. The Maxwell-Boltzmann distribution remains a good approximation provided $3 m_{w} \beta \gtrsim 1$. The Möller velocity can be expressed as

$$
v_{M}=\frac{1}{E E^{\prime}} \sqrt{\left(p \cdot p^{\prime}\right)^{2}-m_{w}^{4}}=\frac{1}{2 E E^{\prime}} \sqrt{s\left(s-4 m_{w}^{2}\right)},
$$

where $E$ and $E^{\prime}$ are the energies of the scattering particles. Note that in the c.m. frame the velocity of the colliding $W$-WIMPs is half the Möller velocity, $v=\sqrt{1-4 m_{w}^{2} / s}=v_{M} / 2$.

For $s \gg m_{\mathrm{f}}$, from (96) and (105) we obtain

$$
\sigma v_{M}=\frac{N_{c}}{8 \pi}\left(\frac{\eta_{\chi} m_{\mathrm{f}} \Delta m}{2\left(s-m_{h}^{2}\right)\left(s-m_{H}^{2}\right)}\right)^{2}\left(s-4 m_{w}^{2}\right) .
$$

We evaluate (103) by expanding $\sigma v_{M}$ around

$$
s=4 E^{2}=\frac{4 m_{w}^{2}}{1-v^{2}} \approx 4 m_{w}^{2}\left(1+v^{2}+\ldots\right)
$$

to obtain a series solution in powers of $v$ of which the leading order term is

$$
\langle\sigma v\rangle \approx \frac{N_{c}}{2 \pi}\left(\frac{\eta_{\chi} m_{\mathrm{f}} m_{w} \Delta m}{2\left(4 m_{w}^{2}-m_{h}^{2}\right)\left(4 m_{w}^{2}-m_{H}^{2}\right)}\right)^{2}\left\langle v^{2}\right\rangle,
$$

where $\left\langle v^{2}\right\rangle$ is the $W$-WIMP thermally averaged velocity. 
All in all, the total average annihilation cross section into SM particles (labelled by subindex $i$ ) is given by

$$
\sum_{\text {fermions }}\left\langle\sigma_{i} v\right\rangle \approx \frac{3}{2 \pi}\left(\frac{\eta_{\chi} m_{b} m_{w} \Delta m}{2\left(4 m_{w}^{2}-m_{H}^{2}\right)}\right)^{2} \frac{\left\langle v^{2}\right\rangle}{\left(4 m_{w}^{2}-m_{h}^{2}\right)^{2}+m_{h}^{2} \Gamma_{h}^{2}},
$$

where we have assumed that the overwhelming contribution into $b \bar{b}$ dominates the process.

Provided the theory is not strongly coupled, (109) is generally a good approximation for relativistic particles, but for low velocities and in the presence of a long-range force (classically, when the potential energy due to the long-range force is comparable to the particles' kinetic energy), the perturbative approach breaks down. In the nonrelativistic limit, the question of how the long-range potential modifies the cross section for short-range interactions can be formulated as a scattering problem in quantum mechanics, with significant modifications to the cross sections occurring when the particle wave functions are no longer well approximated by plane waves (so the Born expansion is not well behaved). The deformation of the wave functions due to a Coulomb potential was calculated by Sommerfeld [96], yielding $\mathrm{a} \sim 1 / v$ enhancement to the cross section for short-range interactions (where the long-range behavior due to the potential can be factorized from the relevant short-range behavior). Along these lines, for low-velocity $\left(v \sim 10^{-3}\right) W$-WIMPs in our Galactic halo, we expect interactions with the $H$ and $h$ fields to enlarge the cross section, as the attractive Yukawa potential,

$$
V_{w}(r)=-\frac{f^{2} \cos ^{2}(\chi)}{4 \pi} \frac{e^{-m_{h} r}}{r}-\frac{f^{2} \sin ^{2}(\chi)}{4 \pi} \frac{e^{-m_{H} r}}{r} \approx-\frac{f^{2}}{4 \pi} \frac{e^{-m_{h} r}}{r} \simeq-\frac{f^{2}}{4 \pi} \frac{1}{r},
$$

causes passing $W$-WIMPS to be drawn toward each other [97].

For $p$-wave scattering, $\left\langle v^{2}\right\rangle \rightarrow\left\langle S(v) v^{2}\right\rangle$, where

$$
S(v) \approx \frac{\pi \tilde{\alpha}}{v} \frac{1}{1-e^{-\pi \tilde{\alpha} / v}}\left(1+\frac{\pi^{2} \tilde{\alpha}^{2}}{4 v^{2}}\right)
$$

is the Sommerfeld enhancement factor in the Coloumb approximation, with $\tilde{\alpha}=f^{2} /(4 \pi)$ [98]. Following [99] we compute the thermally averaged Sommerfeld enhancement factor by approximating $\left(1-e^{-\pi \tilde{\alpha} / v}\right)^{-1}$ with $\tilde{\alpha} \ll 1$

$$
\left\langle S(v) v^{2}\right\rangle \approx 6 x^{-1}+4 \sqrt{\pi} \tilde{\alpha} x^{-1 / 2}+\frac{4 \pi^{2} \tilde{\alpha}^{2}}{3}+\pi^{5 / 2} \tilde{\alpha}^{3} x^{1 / 2}+\frac{\pi^{4} \tilde{\alpha}^{4}}{6} x
$$

where $x=m_{w} / T$. For interactions in the Galactic halo (G.h.), we have $\left\langle v^{2}\right\rangle \sim 10^{-6}$, and therefore the thermally average annihilation cross section into $b \bar{b}$ becomes

$$
\begin{aligned}
\left\langle\sigma_{b} v\right\rangle & \approx \frac{3}{2 \pi}\left(\frac{\eta_{\chi} m_{b} m_{w} \Delta m}{2\left(4 m_{w}^{2}-m_{H}^{2}\right)}\right)^{2} \frac{1}{\left(4 m_{w}^{2}-m_{h}^{2}\right)^{2}+m_{h}^{2} \Gamma_{h}^{2}} \\
& \times \frac{1}{4}\left(6 x_{\text {G.h. }}^{-1}+4 \sqrt{\pi} \tilde{\alpha} x_{\text {G.h. }}^{-1 / 2}+\frac{4 \pi^{2} \tilde{\alpha}^{2}}{3}+\pi^{5 / 2} \tilde{\alpha}^{3} x_{\text {G.h. }}^{1 / 2}+\frac{\pi^{4} \tilde{\alpha}^{4}}{6} x_{\text {G.h. }}\right),
\end{aligned}
$$

with $x_{\text {G.h. }} \approx 3 \times 10^{6}$. 


\section{B. W-WIMP Annihilation into Pairs of Goldstone Bosons}

In addition to the annihilation into SM fermions we must consider the $w \bar{w} \rightarrow 2 \alpha^{\prime}$ annihilation channel. The invariant amplitude for this process is given by

$$
i \mathcal{M}=\frac{2 i f}{v_{r}} \bar{v}(p) u\left(p^{\prime}\right)\left(\frac{\sin ^{2} \chi}{s-m_{H}^{2}}-\frac{\cos ^{2} \chi}{s-m_{h}^{2}}\right) k \cdot k^{\prime} .
$$

We then average over the in state spins to obtain

$$
\frac{1}{4} \sum_{s, s^{\prime}}|\mathcal{M}|^{2}=\frac{f^{2} s^{2}\left[\left(s-m_{h}^{2}\right) \sin ^{2} \chi-\left(s-m_{H}^{2}\right) \cos ^{2} \chi\right]^{2}}{2 v_{r}^{2}\left(s-m_{h}^{2}\right)^{2}\left(s-m_{H}^{2}\right)^{2}}\left(s-4 m_{w}^{2}\right) .
$$

The general expression for the cross section reads

$$
\sigma=\frac{1}{16 \pi \sqrt{s} \sqrt{\left|s-4 m_{w}^{2}\right|}} \frac{f^{4} s^{2}\left[\left(s-m_{h}^{2}\right) \sin ^{2} \chi-\left(s-m_{H}^{2}\right) \cos ^{2} \chi\right]^{2}}{\Delta m^{2}\left(s-m_{h}^{2}\right)^{2}\left(s-m_{H}^{2}\right)^{2}}\left(s-4 m_{w}^{2}\right) .
$$

Using the small angle approximation, i.e. $\cos \chi \approx 1$, we obtain

$$
\sigma \approx \frac{f^{2} s^{2} \sqrt{\left|s-4 m_{w}^{2}\right|}}{16 \pi \sqrt{s}\left(s-m_{h}^{2}\right)^{2}}\left(\frac{f^{2}}{\Delta m^{2}}+\frac{\left(m_{h}^{2}+m_{H}^{2}-2 s\right)}{2\left(s-m_{H}^{2}\right)^{2}} \frac{\eta_{\chi}^{2} v_{\phi}^{2}}{\left(m_{H}^{2}-m_{h}^{2}\right)^{2}}\right) .
$$

Taking a thermal average gives

$$
\left\langle\sigma_{\alpha^{\prime}} v\right\rangle \approx \frac{2 f^{4} m_{w}^{4}}{\pi \Delta m^{2}\left[\left(m_{h}^{2}-4 m_{w}^{2}\right)^{2}+m_{h}^{2} \Gamma_{h}^{2}\right]}\left\langle v^{2}\right\rangle .
$$

If the $W$-WIMPs are highly nonrelativistic we have to correct (117) to account for the Sommerfeld enhancement,

$$
\left\langle\sigma_{\alpha^{\prime}} v\right\rangle \approx \frac{2 f^{4} m_{w}^{4}}{\pi \Delta m^{2}\left[\left(m_{h}^{2}-4 m_{w}^{2}\right)^{2}+m_{h}^{2} \Gamma_{h}^{2}\right]}\left\langle S(v) v^{2}\right\rangle .
$$

\section{W-WIMP Parameter Fits}

The total flux of $\gamma$-rays per solid angle from $W$-WIMP annihilation into SM particles (labelled by subindex $i$ ) is given by

$$
\frac{d \Phi_{\gamma}}{d E_{\gamma}}=\left.\sum_{\text {fermions }} \frac{\left\langle\sigma_{i} v\right\rangle}{2} \frac{\mathcal{J}_{\Delta \Omega}}{J_{0}} \frac{1}{\Delta \Omega_{\mathrm{obs}} m_{w}^{2}} \frac{d N_{\gamma}}{d E_{\gamma}}\right|_{i},
$$

where $\mathcal{J} / J_{0}$ is the normalized integral of mass density squared of the dark matter in the line of sight, $d N_{\gamma} / d E_{\gamma}$ is the $\gamma$-ray spectrum per annihilation into particle species $i, \Delta \Omega_{\text {obs }}$ is the observational solid angle in steradians, and the sum runs over all possible annihilation channels. It is noteworthy that $d \Phi_{\gamma} / d E_{\gamma}$ is the total photon number flux per unit energy per unit steradian for a full sky observation and, when compared to the total photon count of the Fermi-LAT observation with $|b|>10^{\circ}$, must be scaled to the field of view of that observation, $\Delta \Omega_{\mathrm{obs}}=10.4 \mathrm{sr}$. 


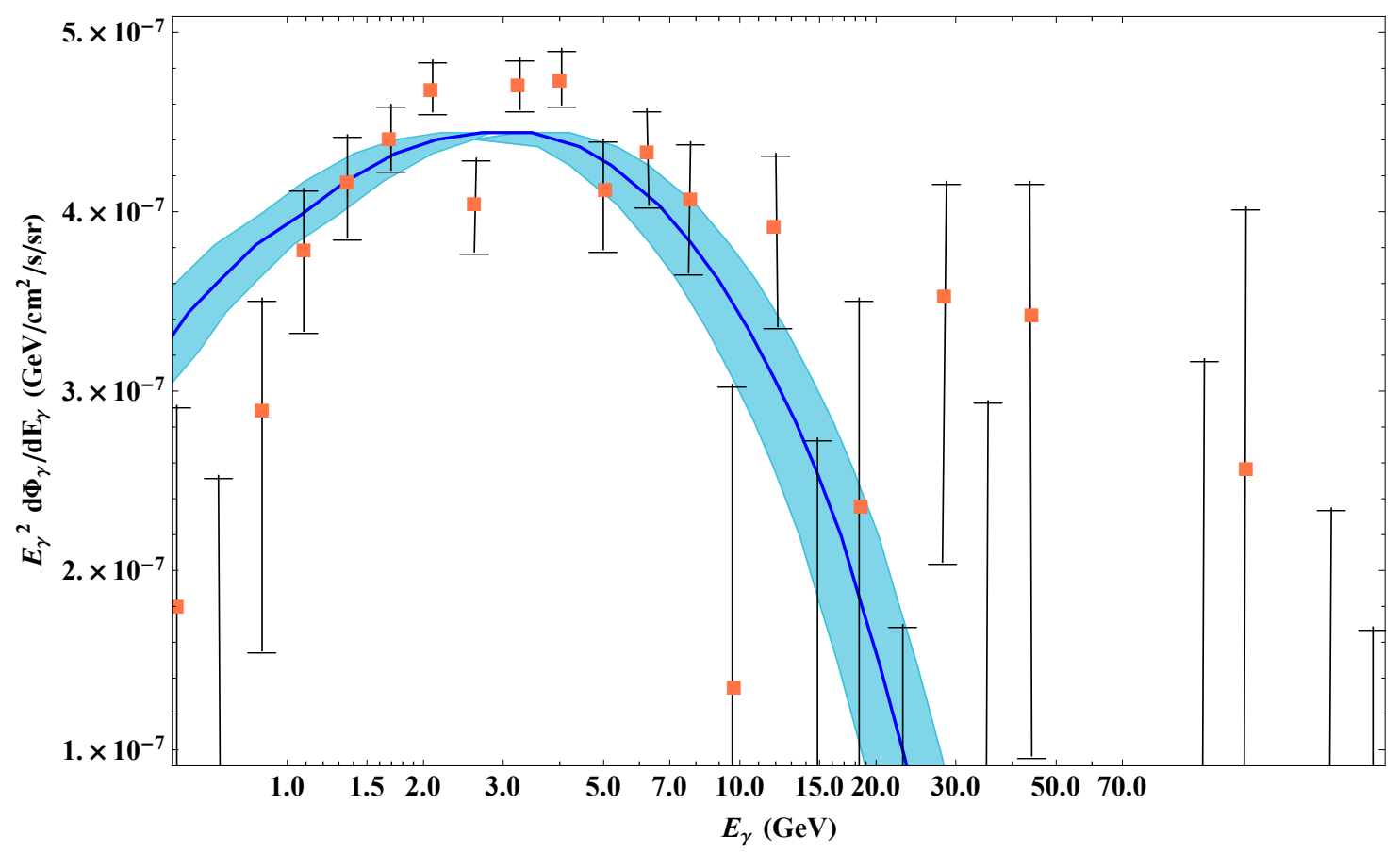

FIG. 2: Comparisons of the observed $\gamma$-ray spectrum of the low-latitude $\left(|b|=10^{\circ}-20^{\circ}\right)$ emission, after subtracting the contribution from inverse Compton scattering to that predicted from $50 \mathrm{GeV}$ $W$-WIMPs annihilating to $b \bar{b}$. We have adopted a generalized NFW profile with an inner slope of $\gamma=1.2$, and normalized the signal to a local density of $0.4 \mathrm{GeV} / \mathrm{cm}^{3}$ and an annihilation cross section of $\left\langle\sigma_{b} v\right\rangle=8 \times 10^{-27} \mathrm{~cm}^{3} / \mathrm{s}$. The band shows the variation in the mass range $45 \mathrm{GeV}<$ $m_{w}<55 \mathrm{GeV}$ for the same normalization. Adapted from Fig. 14 of Ref. [13].

From (109) we see that, for $10 \mathrm{GeV} \lesssim m_{w} \lesssim 50 \mathrm{GeV}$, the dominant annihilation channel is $b \bar{b}$. Annihilation into $c \bar{c}$ and $\tau^{+} \tau^{-}$is suppressed by about 1 order of magnitude. Hereafter, we make the case for a $w$ with a mass of about $50 \mathrm{GeV}$, which annihilates into $b \bar{b}$. The photon flux expected from the Fermi bubbles is shown in Fig. 2. Comparing (2) and (109), we obtain

$$
\begin{aligned}
\left\langle\sigma_{b} v\right\rangle & \approx \frac{3}{2 \pi}\left(\frac{\eta_{\chi} m_{b} m_{w} \Delta m}{2\left(4 m_{w}^{2}-m_{H}^{2}\right)}\right)^{2} \frac{1}{\left(4 m_{w}^{2}-m_{h}^{2}\right)^{2}+m_{h}^{2} \Gamma_{h}^{2}} \frac{1}{4}\left(6 x_{\text {G.h. }}^{-1}+4 \sqrt{\pi} \tilde{\alpha} x_{\text {G.h. }}^{-1 / 2}+\frac{4 \pi^{2} \tilde{\alpha}^{2}}{3}\right. \\
& \left.+\pi^{5 / 2} \tilde{\alpha}^{3} x_{\text {G.h. }}^{1 / 2}+\frac{\pi^{4} \tilde{\alpha}^{4}}{6} x_{\text {G.h. }}\right)=6.7 \times 10^{-10} \mathrm{GeV}^{-2}
\end{aligned}
$$

To be produced thermally in the early Universe in an abundance equal to the measured dark matter density, $\Omega_{\mathrm{DM}} h^{2}=0.1120 \pm 0.0056$ [100], the $50 \mathrm{GeV} w$ particle must have an annihilation cross section of

$$
\sum_{\text {all species }}\left\langle\sigma_{i} v\right\rangle \sim 3 \times 10^{-26} \mathrm{~cm}^{3} / \mathrm{s}=2.5 \times 10^{-9} \mathrm{GeV}^{-2}
$$

when thermally averaged over the process of freeze-out, $x_{\text {f.o. }} \sim 20[101,102]$. It is noteworthy that for $\tilde{\alpha} \lesssim 0.01$ the effect of the Sommerfeld enhancement on the final relic particle 
TABLE II: Best-fit parameters.

\begin{tabular}{cc}
\hline \hline$\Delta m$ & $6000 \mathrm{GeV}$ \\
\hline$m_{h}$ & $98.8 \mathrm{GeV}$ \\
\hline$f$ & 0.34 \\
\hline$\tilde{\alpha}$ & 0.009 \\
\hline$\eta_{\chi}$ & $1.8 \times 10^{-4}$ \\
\hline$\chi$ & 0.049 \\
\hline$\Gamma_{H \rightarrow \text { invisible }}$ & $0.65 \mathrm{MeV}$ \\
\hline$\left\langle\sigma_{\alpha^{\prime}} v\left(x_{\text {f.o. }}\right)\right\rangle$ & $2.7 \times 10^{-26} \mathrm{~cm}^{3} \mathrm{~s}^{-1}$ \\
\hline$\left\langle\sigma_{b} v\left(x_{\text {f.o. }}\right)\right\rangle$ & $0.3 \times 10^{-26} \mathrm{~cm}^{3} \mathrm{~s}^{-1}$ \\
\hline$\left\langle\sigma_{\alpha^{\prime}} v\left(x_{\text {G.h. }}\right)\right\rangle$ & $7.8 \times 10^{-26} \mathrm{~cm}^{3} \mathrm{~s}^{-1}$ \\
\hline \hline
\end{tabular}

abundance is negligible [103, 104]. Herein we will work on the range of the coupling $\tilde{\alpha}$ over which Sommerfeld annihilation can be neglected in the calculation of relic densities.

Because a priori we do not know whether $\left\langle\sigma_{\alpha^{\prime}} v\right\rangle$ or $\left\langle\sigma_{b} v\right\rangle$ dominates the total annihilation cross section at freeze-out, we combine $(109)$ and $(117)$ evaluated at $v\left(x_{\text {f.o. }}\right)$ together with (121) to obtain

$$
\left[\frac{2 f^{4} m_{w}^{4}}{\pi \Delta m^{2}}+\frac{3}{2 \pi}\left(\frac{\eta_{\chi} m_{b} m_{w} \Delta m}{2\left(4 m_{w}^{2}-m_{H}^{2}\right)}\right)^{2}\right] \frac{1}{\left(4 m_{w}^{2}-m_{h}^{2}\right)^{2}+m_{h}^{2} \Gamma_{h}^{2}} \frac{3}{2 x_{\mathrm{f} . \mathrm{o} .}} \sim 2.5 \times 10^{-9} \mathrm{GeV}^{-2}
$$

To determine the allowed region of the parameter space, for $m_{w}=50 \mathrm{GeV}$, we solve (120) and (122) while simultaneously demanding that $\tilde{\alpha} \lesssim 0.01$, and that the upper limit on the invisible decay width for the SM Higgs (34) is not violated by (55). The best-fit parameters are given in Table II, for an example with $\Delta m=6000 \mathrm{GeV}$. We can see that the annihilation into pairs of Goldstone bosons is dominating the $w \bar{w}$ interactions at freeze-out by a factor of about 9 . Precise determination of the parameters is at present hampered by the large uncertainties in the dark matter halo profile. Interestingly, the $W$-WIMP-nucleon cross section is within the reach of the XENON1T experiment [105], providing a strong motivation for the ideas discussed in this section.

Duplicating the procedure described above, we have scanned the mass range of the parameter space that is consistent with Fermi data: $45 \mathrm{GeV}<m_{w}<55 \mathrm{GeV}$; see Fig. 2. Our results are encapsulated in Figs. 3, 4 and 5. In particular, Fig. 3 and 4 display, for $\Delta m=5500 \mathrm{GeV}$, the region of the parameter space of $m_{w}$ vs $\sigma_{w N}$ not yet excluded by current direct detection experiments or the LHC. Future LHC data will either more tightly constrain this parameter space or will turn up evidence for a signal. Note that the region excluded by nonexistence of a solution $\left(\Gamma_{H \rightarrow \text { invisible }} \approx 0.3 \mathrm{MeV}\right)$ up to the current LHC bound will be very tightly constrained after the LHC coming upgrade, assuming no signal appears. In the case that a signal does appear, the combination of relations shown in Figs. 3 and 4 will constrain model parameters providing the XENON1T experiment with the specific cross section required to confirm this model. As an illustration, in Fig. 5 we show contours of constant $\eta_{\chi}$ in the $\Delta m-m_{w}$ plane for the case in which $\mathcal{B}(H \rightarrow$ invisible $)$ saturates the current limit, $\Gamma_{H \rightarrow \text { invisible }}=0.8 \mathrm{MeV}$. The direct detection cross section sampling this subregion of the parameter space varies between $1.8 \times 10^{-46} \mathrm{~cm}^{2}$ and $2.2 \times 10^{-46} \mathrm{~cm}^{2}$, with an average of $1.9 \times 10^{-46} \mathrm{~cm}^{2}$. 


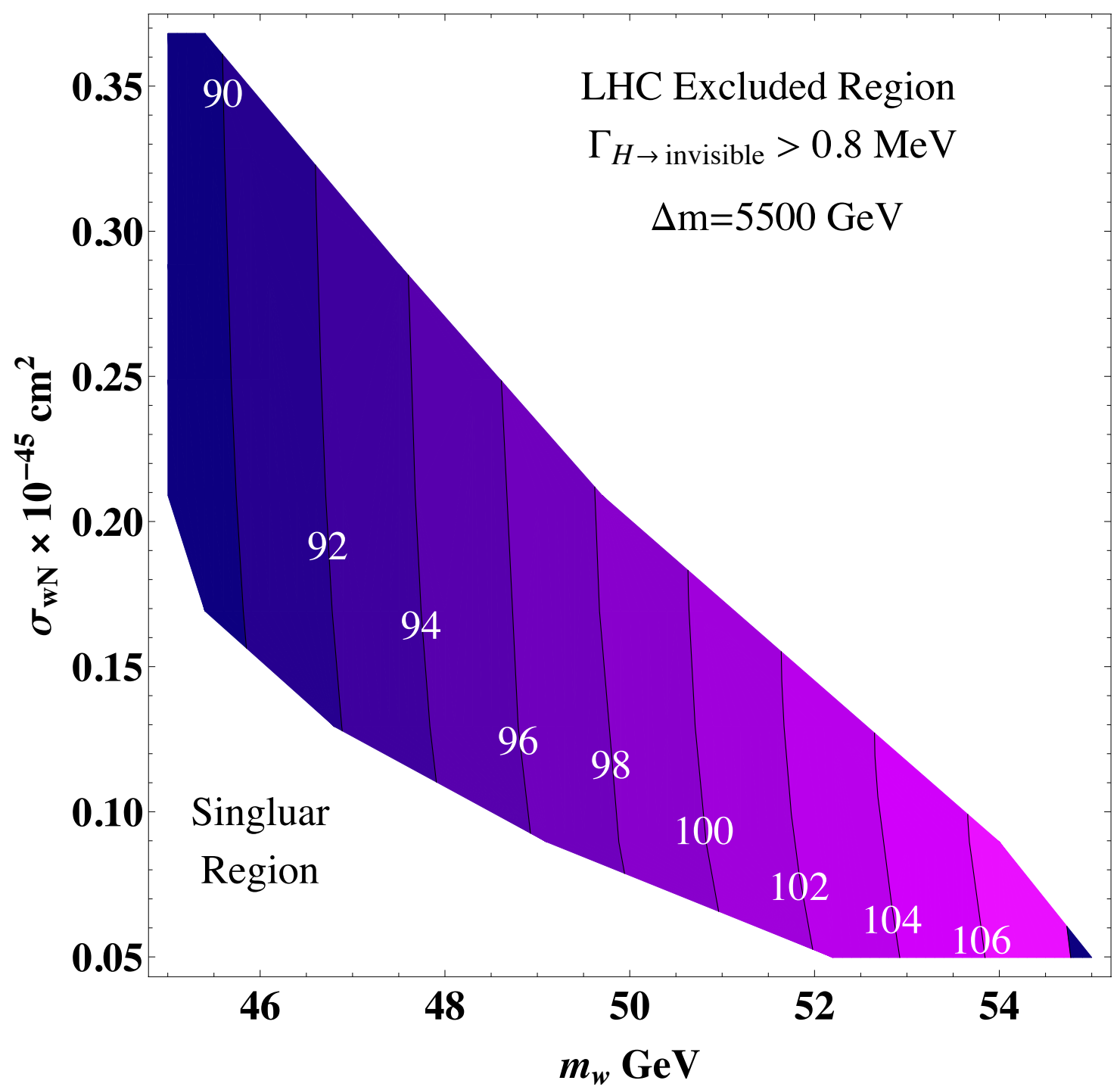

FIG. 3: Contours of constant $m_{h} / \mathrm{GeV}$ in the $\sigma_{w N}-m_{w}$ plane. The contours satisfy Fermi data, the relic density requirement, and the LHC bound $\mathcal{B}(H \rightarrow$ invisible $)$. We have required $\tilde{\alpha} \lesssim 0.01$ and taken $\Delta m=5500 \mathrm{GeV}$.

\section{VII. $W$-WIMP INTERPRETATION FOR HINTS OF LIGHT DARK MATTER}

Signals broadly compatible with $\sim 10 \mathrm{GeV}$ dark matter have been observed in four direct detection experiments: DAMA/LIBRA [23], CoGeNT [24, 25], CRESST [26], and CDMS-II [27]. In this section we explore the compatibility with one particular region of the $W$-WIMP parameter space. The features of this region of the parameter space has bearing on the evidence for extrarelativistic degrees of freedom at the CMB epoch.

In order to elaborate on the case for $m_{w} \sim 10 \mathrm{GeV}$, we consider $m_{h} \approx 500 \mathrm{MeV}$ and $\eta_{\chi} \approx 0.005$. Substituting these values in (67), it is straightforward to see that to comply with the elastic cross section signal reported by the CDMS Collaboration [27], we must set $\Delta m \approx 1.75 \mathrm{GeV}$. This in turn determines via (109) a thermal average annihilation cross section into quarks: $\left\langle\sigma_{b} v\left(x_{\text {G.h. }}\right)\right\rangle \approx 1.3 \times 10^{-39} \mathrm{~cm}^{3} \mathrm{~s}^{-1}$. Note that this is more than 10 orders of magnitude smaller than current limits on light dark matter from antiproton 


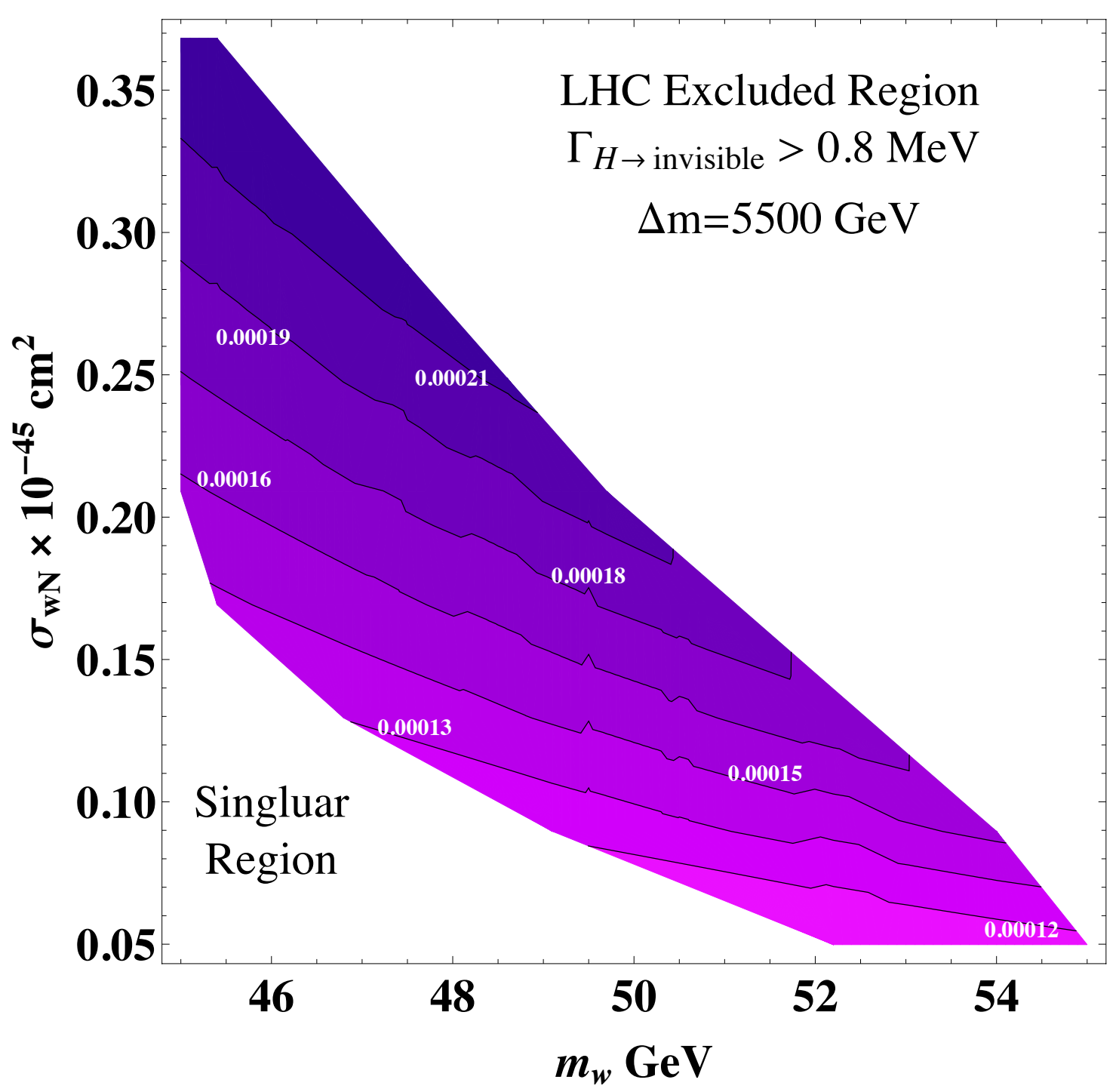

FIG. 4: Contours of constant $\eta_{\chi}$ in the $\sigma_{w N}-m_{w}$ plane. Again the contours satisfy Fermi data, the relic density requirement, and the LHC bound $\mathcal{B}(H \rightarrow$ invisible $)$. We have required $\tilde{\alpha} \lesssim 0.01$ and taken $\Delta m=5500 \mathrm{GeV}$.

data [106-108].

The observed dark matter density is obtained again through dominant $W$-WIMP annihilation into the hidden sector. To demonstrate this point, we must first compute the $w \bar{w} \rightarrow 2 h$ annihilation cross section, as this channel is now open. We consider the relevant terms of $(32)$,

$$
\frac{f \cos \chi}{2} h \bar{\psi}_{-} \psi_{-}+\frac{f \sin \chi}{2} H \bar{\psi}_{-} \psi_{-}
$$

as well as the relevant terms of the scalar potential

$$
\mathcal{V} \approx \cdots-\frac{m_{h}^{2}}{2 v_{r}} h^{3}-\frac{\eta_{\chi} v_{\phi}}{2}\left(\frac{m_{H}^{2}+2 m_{h}^{2}}{m_{H}^{2}-m_{h}^{2}}\right) H h^{2}
$$




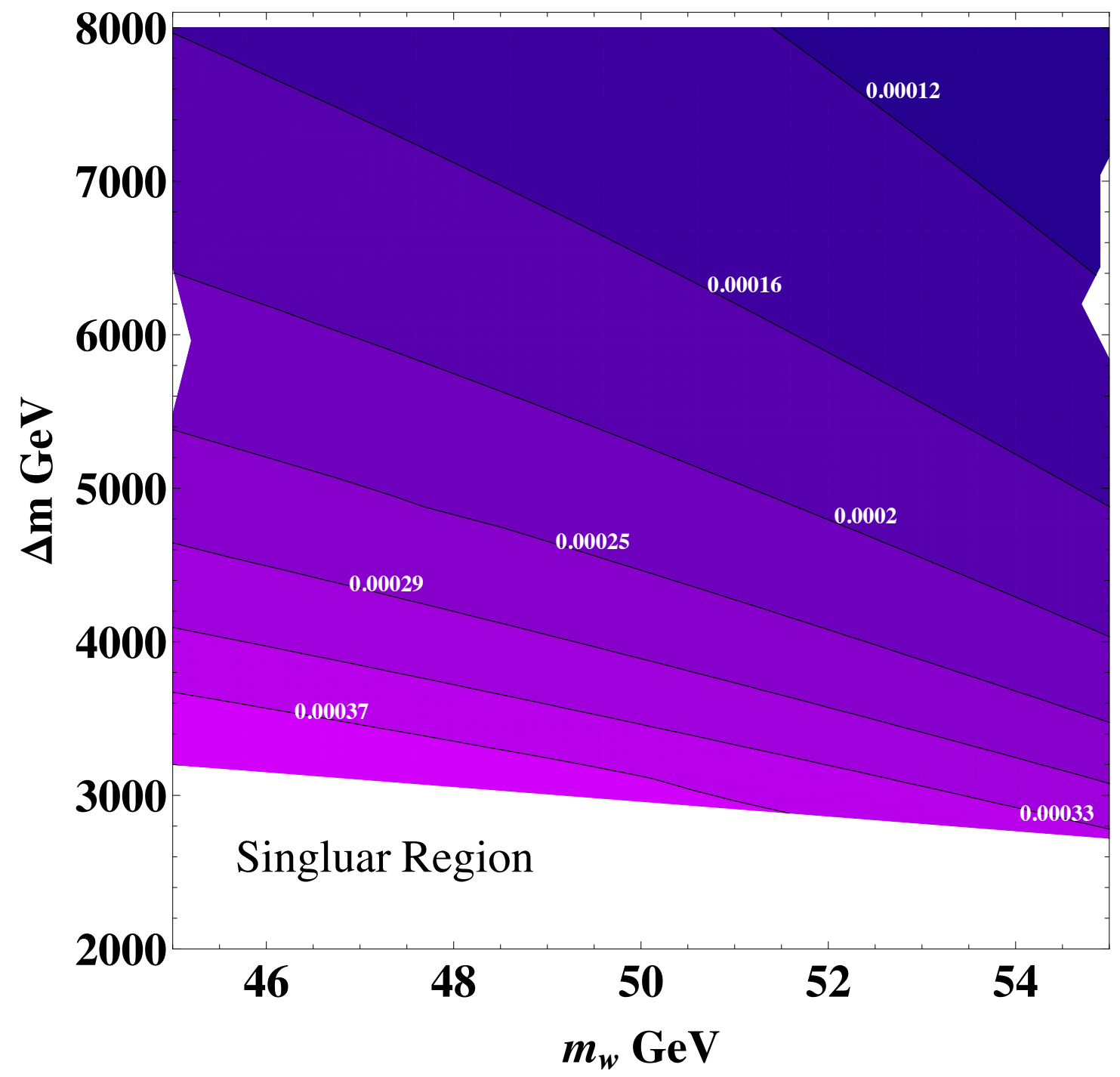

FIG. 5: Contours of constant $\eta_{\chi}$ in the $\Delta m-m_{w}$ plane. The contours satisfy Fermi data, the relic density requirement, and saturates the LHC bound $\mathcal{B}(H \rightarrow$ invisible $)$. We have required $\tilde{\alpha} \lesssim 0.01$ and we have verified that the XENON-100 upper limit [34] is not violated.

together this gives the total reaction matrix element

$$
\begin{aligned}
\mathcal{M} & =i f \bar{v}(p) u\left(p^{\prime}\right) \frac{i}{s-m_{h}^{2}}\left(\frac{-i 3 ! m_{h}^{2}}{2 v_{r}}\right)+i f \frac{\eta_{\chi} v_{r} v_{\phi}}{m_{H}^{2}-m_{h}^{2}} \bar{v}(p) u\left(p^{\prime}\right) \frac{i}{s-m_{H}^{2}} \\
& \times\left(\frac{-i \eta_{\chi} v_{\phi}\left(m_{H}^{2}+2 m_{h}^{2}\right)}{m_{H}^{2}-m_{h}^{2}}\right) .
\end{aligned}
$$

Assuming $\eta_{\chi} \ll 1$ and $m_{h} \ll m_{H}$, we arrive at a manageable form of the spin-averaged 
$w \bar{w} \rightarrow 2 h$ amplitude

$$
\begin{aligned}
\frac{1}{4} \sum_{\text {spins }}|\mathcal{M}|^{2} & \approx f^{2}\left(\frac{9 m_{h}^{4}}{v_{r}^{2}\left(s-m_{h}^{2}\right)^{2}}+\frac{6 m_{h}^{2} \eta_{\chi}^{2} v_{\phi}^{2}\left(m_{H}^{2}+2 m_{h}^{2}\right)}{m_{H}^{4}\left(s-m_{h}^{2}\right)\left(s-m_{H}^{2}\right)}\right)\left(p \cdot p^{\prime}-m_{w}^{2}\right), \\
& \approx f^{2}\left(\frac{18 f^{2} m_{h}^{4}}{\Delta m^{2}\left(s-m_{h}^{2}\right)^{2}}+\frac{3 m_{h}^{2} \eta_{\chi}^{2} v_{\phi}^{2}}{m_{H}^{2}\left(s-m_{h}^{2}\right)\left(s-m_{H}^{2}\right)}\right)\left(s-4 m_{w}^{2}\right),
\end{aligned}
$$

and the scattering cross section

$$
\sigma \approx \frac{f^{2}}{32 \pi s} \sqrt{\frac{s-4 m_{h}^{2}}{s-4 m_{w}^{2}}}\left(\frac{18 f^{2} m_{h}^{4}}{\Delta m^{2}\left(s-m_{h}^{2}\right)^{2}}+\frac{3 m_{h}^{2} \eta_{\chi}^{2} v_{\phi}^{2}}{m_{H}^{2}\left(s-m_{h}^{2}\right)\left(s-m_{H}^{2}\right)}\right)\left(s-4 m_{w}^{2}\right) .
$$

We take the thermal average in the low temperature limit, that is $T \ll m_{w}$,

$$
\left\langle\sigma_{h} v\right\rangle \approx\left(\frac{9 f^{4} m_{h}^{4}}{8 \pi \Delta m^{2}\left(m_{h}^{2}-4 m_{w}^{2}\right)^{2}}+\frac{3 f^{2} v_{\phi}^{2} \eta_{\chi}^{2} m_{h}^{2}}{16 \pi m_{H}^{4}\left(m_{h}^{2}-4 m_{w}^{2}\right)}\right)\left\langle v^{2}\right\rangle .
$$

By demanding the total annihilation cross section to comply with the relic density requirement [102] we obtain

$$
\left\langle\sigma_{\alpha^{\prime}} v\right\rangle_{+}\left\langle\sigma_{h} v\right\rangle+\sum_{\text {fermions }}\left\langle\sigma_{i} v\right\rangle \sim 3 \times 10^{-26} \mathrm{~cm}^{3} / s,
$$

and so

$$
f \approx 0.04 \text {. }
$$

As a final check we ensure that the LHC upper limit on the hidden decay width of the Higgs is satisfied; taking note that the decay channel $H \rightarrow \bar{\psi}_{+} \psi_{+}$is now open, we have

$$
\frac{\eta_{\chi}^{2} v_{\phi}^{2}}{16 \pi m_{H}}+\frac{\eta_{\chi}^{2} \Delta m^{2} v_{\phi}^{2}}{32 \pi m_{H}^{3}}=0.24 \mathrm{MeV}<0.8 \mathrm{MeV}
$$

In Fig. 6 we exhibit the range of parameters consistent with the 95\% C.L. upper limit on $\mathcal{B}(H \rightarrow$ invisible) [51-54] together with possible signal regions associated with data from CDMS-II [27]. For $m_{w}=10 \mathrm{GeV}$, the best-fit intervals at the $68 \%$ C.L. and the $90 \%$ C.L. are $3 \times 10^{-42}<\sigma_{w N} / \mathrm{cm}^{2}<2.5 \times 10^{-41}$ and $2 \times 10^{-42}<\sigma_{w N} / \mathrm{cm}^{2}<3 \times 10^{-41}$, respectively. The horizontal lines preserve the constant $\eta_{\chi} / m_{h}$ ratio that allows decoupling of $\alpha^{\prime}$ at $T \approx m_{\mu}$, yielding $N_{\text {eff }}=3.39$.

In summary, we have shown that $W$-WIMPs of about $10 \mathrm{GeV}$ can simultaneously explain the observed relic density and the possible signals observed by direct detection experiments, while avoiding limits from indirect detection experiments. In the near future, the Large Underground Xenon (LUX) dark matter experiment [109] will collect enough statistics to probe the $\sim 10 \mathrm{GeV}$ dark matter hypothesis. Concurrent with LUX observations will be precise measurements of the Higgs branching fractions by the LHC ATLAS and CMS experiments (operating at $\sqrt{s}=14 \mathrm{TeV}$ ). This new arsenal of data, when combined with observations the Phased IceCube Next Generation Upgrade (PINGU) [110], will have the potential to single out this distinctive Higgs portal light dark matter model. ${ }^{4}$

${ }^{4}$ Since the annihilation rate into SM particles is largely suppressed compared to annihilations into the hidden sector, this particular model predicts null results at PINGU. 


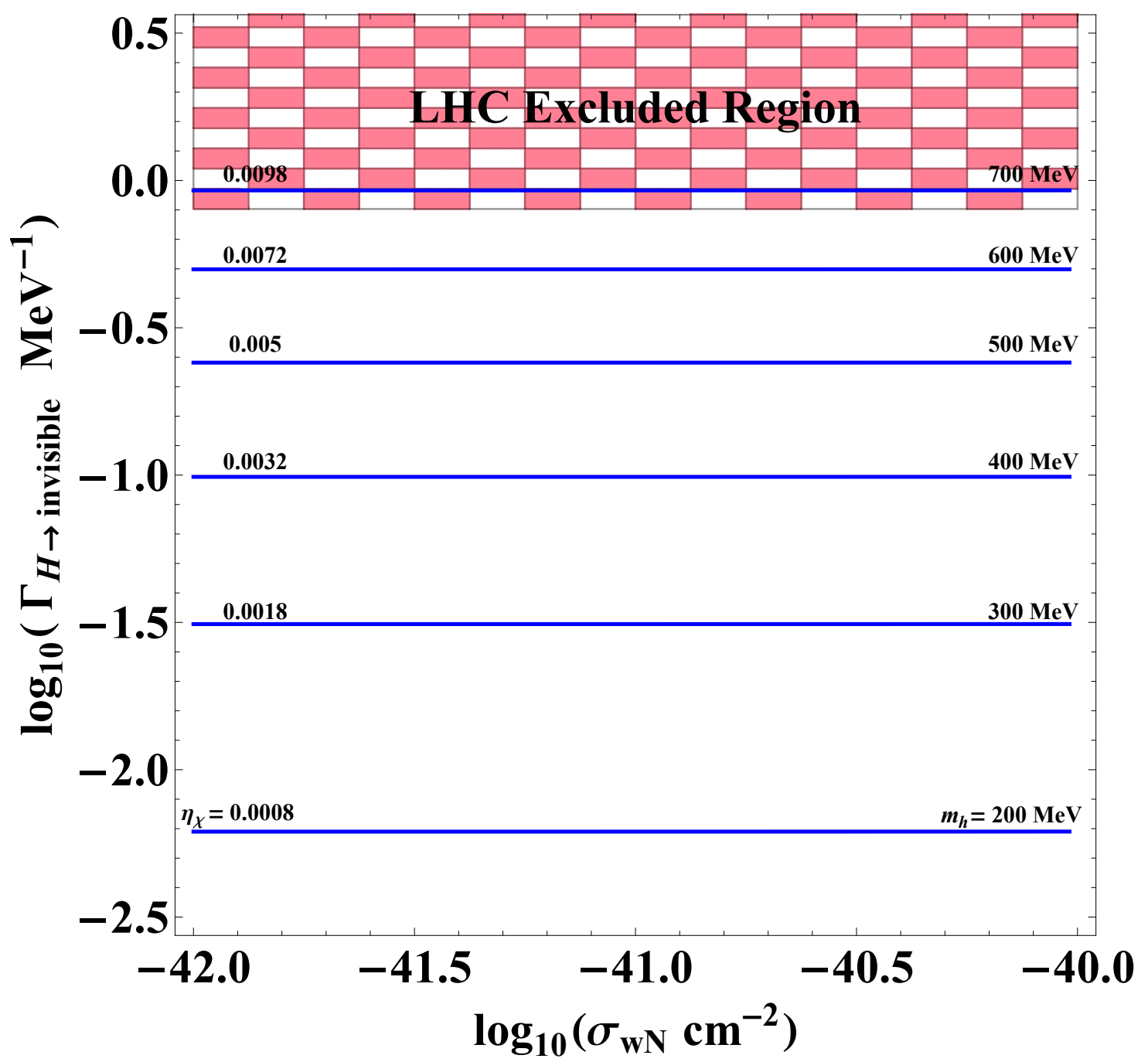

FIG. 6: $\Gamma_{H \rightarrow \text { invisible }}$ for varying values of $\sigma_{w N}$. The plotted values are nearly constant as the terms from $\Gamma_{H \rightarrow \alpha, h}$ dominate the decay width, and thus there is weak dependence on the direct detection cross section. For varying values of $m_{h}$ we adjust the value of $\eta_{\chi}$ so that the Goldstone bosons decouple from the primordial plasma at $k_{B} T \approx m_{\mu}$, yielding $N_{\text {eff }}=3.39$. For $200 \mathrm{MeV} \leq m_{h} \leq$ $700 \mathrm{MeV}$, the Higgs decay width into the hidden sector varies between $(0.006-0.92) \mathrm{MeV}$. The constant $\eta_{\chi} / m_{h}$ contours shown here are independent of $m_{w}$, and therefore span the mass range $7 \mathrm{GeV} \lesssim m_{w} / \mathrm{GeV} \lesssim 10$

\section{CONCLUSIONS}

$\gamma$-ray data in the $1-100 \mathrm{GeV}$ range from Fermi show a new unexpected feature of the Milky Way: two huge spheroidal structures called Fermi bubbles, extending up to about $10 \mathrm{kpc}\left(50^{\circ}\right)$ out of the $\mathrm{GC}$ on either side of the Galactic disk. Intriguingly, the bubbles coincide spatially with the WMAP haze in microwave and the thermal x-ray emission seen by ROSAT. There is a general consensus in that far from the GC the $\gamma$-rays observed by Fermi originate from inverse Compton scattering of photons from the interstellar radiation field by the same hard electron population that produces the haze via synchrotron. Very 
recently, a second component of $\gamma$-ray emission from the low-latitude regions of the Fermi bubbles has been identified. The spectral shape of this new component is consistent with that expected from an approximately $50 \mathrm{GeV}$ dark matter particle annihilating into $b \bar{b}$, with a normalization corresponding to $\left\langle\sigma_{b} v\right\rangle \sim 8 \times 10^{-27} \mathrm{~cm}^{3} / \mathrm{s}$, or else with $10 \mathrm{GeV}$ dark matter particle annihilating dominantly into $\tau^{+} \tau^{-}$, with a normalization corresponding to $\left\langle\sigma_{\tau} v\right\rangle \sim 2 \times 10^{-27} \mathrm{~cm}^{3} / \mathrm{s}$.

We have shown that $W$-WIMPs (with $m_{w} \approx 50 \mathrm{GeV}$ ) are capable of accommodating the desired effective annihilation into $b \bar{b}$. We have also demonstrated that the thermal cross section required to account for the relic dark matter abundance can easily be obtained if $w \bar{w} \rightarrow 2 \alpha$ is the dominant annihilation channel. However, given that the Goldstone bosons would decouple at $5 \mathrm{GeV}$ (i.e. in the very early Universe), the contribution to the effective number of neutrinos for the described parameter space is negligible and thus cannot explain the evidence for dark radiation (assuming there exist no common systematic uncertainties in the measurements of the Hubble parameter). In the near future, the upgraded LHC together with the new XENON1T experiment will further whittle down the parameter space, or else make a discovery.

If $m_{w} \approx 10 \mathrm{GeV}$, Weinberg's hidden sector does not provide a viable explanation of the Fermi bubbles since the $W$-WIMP annihilation would be dominated by $b \bar{b}$ rather than $\tau^{+} \tau^{-}$. However, there remains an interesting region of the parameter space which can account for the alleged signals recently reported by direct detection experiments. In this region, the Goldstone bosons decouple from the primordial plasma near the $100 \mathrm{MeV}$ temperature, consistent with the two measurements of the effective number of neutrinos reported by the Planck Collaboration. In this region of the parameter space, $W$-WIMP annihilation into Goldstone bosons is also sufficient for consistency of the observed dark matter abundance. Furthermore, future LHC measurements will further constrain this sector of the Higgs portal (or bettter, find a signal), while LUX will close the deliberations on the alleged direct signals.

In closing, we note that the Fermi bubble production mechanism proposed in this paper is also applicable to more general "hidden valleys" [111, 112]. The mixing between a more elaborate hidden sector and the visible sector modulates the rate of annihilation into SM fields and hidden Goldstone bosons.

\section{Acknowledgments}

We thank Dan Hooper, Tom Paul, Diego Torres, and Tom Weiler for some valuable discussion. L.A.A. and B.J.V. are supported by the U.S. National Science Foundation (NSF) under CAREER Grant PHY-1053663.

[1] R. Schabinger and J. D. Wells, Phys. Rev. D 72, 093007 (2005) [hep-ph/0509209].

[2] B. Patt and F. Wilczek, hep-ph/0605188.

[3] V. Barger, P. Langacker, M. McCaskey, M. J. Ramsey-Musolf and G. Shaughnessy, Phys. Rev. D 77, 035005 (2008) [arXiv:0706.4311 [hep-ph]].

[4] V. Barger, P. Langacker, M. McCaskey, M. Ramsey-Musolf and G. Shaughnessy, Phys. Rev. D 79, 015018 (2009) [arXiv:0811.0393 [hep-ph]]. 
[5] S. Andreas, C. Arina, T. Hambye, F. -S. Ling and M. H. G. Tytgat, Phys. Rev. D 82, 043522 (2010) [arXiv:1003.2595 [hep-ph]].

[6] S. Bock, R. Lafaye, T. Plehn, M. Rauch, D. Zerwas and P. M. Zerwas, Phys. Lett. B 694, 44 (2010) [arXiv:1007.2645 [hep-ph]].

[7] H. E. Logan, Phys. Rev. D 83, 035022 (2011) [arXiv:1010.4214 [hep-ph]].

[8] C. Englert, T. Plehn, D. Zerwas and P. M. Zerwas, Phys. Lett. B 703, 298 (2011) [arXiv:1106.3097 [hep-ph]].

[9] L. Goodenough and D. Hooper, arXiv:0910.2998 [hep-ph].

[10] D. Hooper and L. Goodenough, Phys. Lett. B 697, 412 (2011) [arXiv:1010.2752 [hep-ph]].

[11] D. Hooper and T. Linden, Phys. Rev. D 84, 123005 (2011) [arXiv:1110.0006 [astro-ph.HE]].

[12] K. N. Abazajian and M. Kaplinghat, Phys. Rev. D 86, 083511 (2012) [arXiv:1207.6047 [astro-ph.HE]].

[13] D. Hooper and T. R. Slatyer, Phys. Dark Univ. (to be published) [arXiv:1302.6589 [astroph.HE]].

[14] G. Dobler, D. P. Finkbeiner, I. Cholis, T. R. Slatyer and N. Weiner, Astrophys. J. 717, 825 (2010) [arXiv:0910.4583 [astro-ph.HE]].

[15] M. Su, T. R. Slatyer and D. P. Finkbeiner, Astrophys. J. 724, 1044 (2010) [arXiv:1005.5480 [astro-ph.HE]].

[16] D. P. Finkbeiner, Astrophys. J. 614, 186 (2004) [astro-ph/0311547].

[17] P. A. R. Ade et al. [Planck Collaboration], arXiv:1208.5483 [astro-ph.GA].

[18] S. L. Snowden, R. Egger, M. J. Freyberg, D. McCammon, P. P. Plucinsky, W. T. Sanders, J. H. M. Schmitt and J. Truemper et al., Astrophys. J. 485, 125 (1997).

[19] P. Mertsch and S. Sarkar, Phys. Rev. Lett. 107, 091101 (2011) [arXiv:1104.3585 [astroph.HE]].

[20] D. Hooper, I. Cholis, T. Linden, J. Siegal-Gaskins and T. Slatyer, arXiv:1305.0830 [astroph.HE].

[21] F. Iocco, M. Pato, G. Bertone and P. Jetzer, JCAP 1111, 029 (2011) [arXiv:1107.5810 [astro-ph.GA]].

[22] D. Hooper, Phys. Dark Univ. 1, 1 (2012) [arXiv:1201.1303 [astro-ph.CO]].

[23] R. Bernabei et al., Eur. Phys. J. C 6739 (2010) [arXiv:1002.1028 [astro-ph.GA]].

[24] C. E. Aalseth et al. [CoGeNT Collaboration], Phys. Rev. Lett. 106, 131301 (2011) [arXiv:1002.4703 [astro-ph.CO]].

[25] C. E. Aalseth et al. [CoGeNT Collaboration], Phys. Rev. Lett. 107 (2011) 141301 [arXiv:1106.0650 [astro-ph.CO]].

[26] G. Angloher et al., Eur. Phys. J. C 72, 1971 (2012) [arXiv:1109.0702 [astro-ph.CO]].

[27] R. Agnese et al. [CDMS Collaboration], [arXiv:1304.4279 [hep-ex]].

[28] A. L. Fitzpatrick, D. Hooper and K. M. Zurek, Phys. Rev. D 81, 115005 (2010) [arXiv:1003.0014 [hep-ph]].

[29] S. Chang, J. Liu, A. Pierce, N. Weiner and I. Yavin, JCAP 1008, 018 (2010) [arXiv:1004.0697 [hep-ph]].

[30] D. Hooper, J. I. Collar, J. Hall, D. McKinsey and C. Kelso, Phys. Rev. D 82, 123509 (2010) [arXiv:1007.1005 [hep-ph]].

[31] M. R. Buckley, D. Hooper and T. M. P. Tait, Phys. Lett. B 702, 216 (2011) [arXiv:1011.1499 [hep-ph]].

[32] D. Hooper, F. Petriello, K. M. Zurek and M. Kamionkowski, Phys. Rev. D 79, 015010 (2009) [arXiv:0808.2464 [hep-ph]]. 
[33] R. Kappl and M. W. Winkler, Nucl. Phys. B 850, 505 (2011) [arXiv:1104.0679 [hep-ph]].

[34] E. Aprile et al. [XENON100 Collaboration], Phys. Rev. Lett. 107, 131302 (2011) [arXiv:1104.2549 [astro-ph.CO]].

[35] D. Hooper, arXiv:1306.1790 [hep-ph].

[36] M. Ackermann et al. [Fermi-LAT Collaboration], Phys. Rev. Lett. 107, 241302 (2011) [arXiv:1108.3546 [astro-ph.HE]].

[37] A. Geringer-Sameth and S. M. Koushiappas, Phys. Rev. Lett. 107, 241303 (2011) [arXiv:1108.2914 [astro-ph.CO]].

[38] M. Ackermann et al., JCAP 1005, 025 (2010) [arXiv:1002.2239 [astro-ph.CO]].

[39] K. N. Abazajian, P. Agrawal, Z. Chacko and C. Kilic, JCAP 1011, 041 (2010) [arXiv:1002.3820 [astro-ph.HE]].

[40] A. A. Abdo et al. [Fermi-LAT Collaboration], JCAP 1004, 014 (2010) [arXiv:1002.4415 [astro-ph.CO]].

[41] S. Weinberg, Phys. Rev. Lett. 110, 241301 (2013) [arXiv:1305.1971 [astro-ph.CO]].

[42] P. Sikivie, Phys. Rev. Lett. 48, 1156 (1982).

[43] A. Vilenkin and A. E. Everett, Phys. Rev. Lett. 48, 1867 (1982).

[44] C. T. Hill, D. N. Schramm and L. M. Widrow, FERMILAB-PUB-89-166-T.

[45] A. D. Linde and D. H. Lyth, Phys. Lett. B 246, 353 (1990).

[46] M. S. Turner and F. Wilczek, Phys. Rev. Lett. 66, 5 (1991).

[47] G. R. Dvali, Phys. Lett. B 265, 64 (1991).

[48] T. Hiramatsu, M. Kawasaki, K. 'i. Saikawa and T. Sekiguchi, JCAP 1301, 001 (2013) [arXiv:1207.3166 [hep-ph]].

[49] G. Aad et al. [ATLAS Collaboration], Phys. Lett. B 716, 1 (2012) [arXiv:1207.7214 [hep-ex]].

[50] S. Chatrchyan et al. [CMS Collaboration], Phys. Lett. B 716, 30 (2012) [arXiv:1207.7235 [hep-ex]].

[51] J. R. Espinosa, M. Muhlleitner, C. Grojean and M. Trott, JHEP 1209, 126 (2012) [arXiv:1205.6790 [hep-ph]].

[52] K. Cheung, J. S. Lee and P. -Y. Tseng, JHEP 1305, 134 (2013) [arXiv:1302.3794 [hep-ph]].

[53] P. P. Giardino, K. Kannike, I. Masina, M. Raidal and A. Strumia, arXiv:1303.3570 [hep-ph].

[54] J. Ellis and T. You, arXiv:1303.3879 [hep-ph].

[55] M. Srednicki, Quantum Field Theory, (Cambridge, UK: University Press, 2007).

[56] R. Bernabei, P. Belli, F. Montecchia, W. Di Nicolantonio, A. Incicchitti, D. Prosperi, C. Bacci and C. J. Dai et al., Phys. Lett. B 424195 (1998).

[57] A. K. Drukier, K. Freese and D. N. Spergel, Phys. Rev. D 333495 (1986).

[58] K. Freese, J. A. Frieman and A. Gould, Phys. Rev. D 373388 (1988).

[59] R. Bernabei et al. [DAMA Collaboration], Eur. Phys. J. C 56333 (2008) [arXiv:0804.2741 [astro-ph]].

[60] F. Petriello and K. M. Zurek, JHEP 0809, 047 (2008) [arXiv:0806.3989 [hep-ph]].

[61] J. Angle et al. [XENON10 Collaboration], Phys. Rev. Lett. 107, 051301 (2011) [arXiv:1104.3088 [astro-ph.CO]].

[62] J. I. Collar, arXiv:1010.5187 [astro-ph.IM].

[63] J. I. Collar, arXiv:1106.0653 [astro-ph.CO].

[64] J. R. Ellis, A. Ferstl and K. A. Olive, Phys. Lett. B 481, 304 (2000) [hep-ph/0001005].

[65] M. Beltran, D. Hooper, E. W. Kolb and Z. C. Krusberg, Phys. Rev. D 80, 043509 (2009) [arXiv:0808.3384 [hep-ph]].

[66] G. Steigman, D.N. Schramm and J.E. Gunn, Phys. Lett. B 66, 202 (1977). 
[67] R. H. Cyburt, B. D. Fields, K. A. Olive and E. Skillman, Astropart. Phys. 23, 313 (2005) [astro-ph/0408033].

[68] V. Simha and G. Steigman, JCAP 0806, 016 (2008) [arXiv:0803.3465 [astro-ph]].

[69] Y. I. Izotov and T. X. Thuan, Astrophys. J. 710, L67 (2010) [arXiv:1001.4440 [astro-ph.CO]].

[70] G. Mangano and P. D. Serpico, Phys. Lett. B 701, 296 (2011) [arXiv:1103.1261 [astroph.CO]].

[71] M. Pettini and R. Cooke, arXiv:1205.3785 [astro-ph.CO].

[72] G. Steigman, Adv. High Energy Phys. 2012, 268321 (2012) [arXiv:1208.0032 [hep-ph]].

[73] E. Komatsu et al. [WMAP Collaboration], Astrophys. J. Suppl. 192, 18 (2011) [arXiv:1001.4538 [astro-ph.CO]].

[74] M. C. Gonzalez-Garcia, M. Maltoni and J. Salvado, JHEP 1008, 117 (2010) [arXiv:1006.3795 [hep-ph]].

[75] J. Dunkley, R. Hlozek, J. Sievers, V. Acquaviva, P. A. R. Ade, P. Aguirre, M. Amiri and J. W. Appel et al., Astrophys. J. 739, 52 (2011) [arXiv:1009.0866 [astro-ph.CO]].

[76] Z. Hou, R. Keisler, L. Knox, M. Millea and C. Reichardt, arXiv:1104.2333 [astro-ph.CO].

[77] R. Keisler, C. L. Reichardt, K. A. Aird, B. A. Benson, L. E. Bleem, J. E. Carlstrom, C. L. Chang and H. M. Cho et al., Astrophys. J. 743, 28 (2011) [arXiv:1105.3182 [astroph.CO]].

[78] M. Moresco, A. Cimatti, R. Jimenez, L. Pozzetti, G. Zamorani, M. Bolzonella, J. Dunlop and F. Lamareille et al., JCAP 1208, 006 (2012) [arXiv:1201.3609 [astro-ph.CO]].

[79] G. Hinshaw, D. Larson, E. Komatsu, D. N. Spergel, C. L. Bennett, J. Dunkley, M. R. Nolta and M. Halpern et al., arXiv:1212.5226 [astro-ph.CO].

[80] Z. Hou, C. L. Reichardt, K. T. Story, B. Follin, R. Keisler, K. A. Aird, B. A. Benson and L. E. Bleem et al., arXiv:1212.6267 [astro-ph.CO].

[81] E. Di Valentino, S. Galli, M. Lattanzi, A. Melchiorri, P. Natoli, L. Pagano and N. Said, arXiv:1301.7343 [astro-ph.CO].

[82] E. Calabrese, R. A. Hlozek, N. Battaglia, E. S. Battistelli, J. R. Bond, J. Chluba, D. Crichton and S. Das et al., arXiv:1302.1841 [astro-ph.CO].

[83] M. Benetti, M. Gerbino, W. H. Kinney, E. W. Kolb, M. Lattanzi, A. Melchiorri, L. Pagano and A. Riotto, arXiv:1303.4317 [astro-ph.CO].

[84] P. A. R. Ade et al. [Planck Collaboration], arXiv:1303.5076 [astro-ph.CO].

[85] G. Mangano, G. Miele, S. Pastor, T. Pinto, O. Pisanti and P. D. Serpico, Nucl. Phys. B 729, 221 (2005) [hep-ph/0506164].

[86] A. G. Riess et al., Astrophys. J. 730, 119 (2011) [Erratum ibid. 732, 129 (2011)] [arXiv:1103.2976 [astro-ph.CO]].

[87] W. J. Percival et al. [SDSS Collaboration], Mon. Not. Roy. Astron. Soc. 401, 2148 (2010) [arXiv:0907.1660 [astro-ph.CO]].

[88] E. W. Kolb and M. S. Turner, Front. Phys. 69, 1 (1990).

[89] P. Gondolo and G. Gelmini, Nucl. Phys. B 360, 145 (1991).

[90] T. J. Weiler, AIP Conf. Proc. 1534, 165 (2012) [arXiv:1301.0021 [hep-ph]].

[91] L. A. Anchordoqui and H. Goldberg, Phys. Rev. Lett. 108, 081805 (2012) [arXiv:1111.7264 [hep-ph]].

[92] L. A. Anchordoqui, I. Antoniadis, H. Goldberg, X. Huang, D. Lust, T. R. Taylor and B. Vlcek, Phys. Rev. D 86, 066004 (2012) [arXiv:1206.2537 [hep-ph]].

[93] A. Solaguren-Beascoa and M. C. Gonzalez-Garcia, Phys. Lett. B 719, 121 (2013) [arXiv:1210.6350 [hep-ph]]. 
[94] L. A. Anchordoqui, H. Goldberg and G. Steigman, Phys. Lett. B 718, 1162 (2013) [arXiv:1211.0186 [hep-ph]].

[95] L. A. Anchordoqui, H. Goldberg and B. Vlcek, arXiv:1305.0146 [astro-ph.CO].

[96] A. Sommerfeld, Annalen der Physik 403, 257 (1931).

[97] N. Arkani-Hamed, D. P. Finkbeiner, T. R. Slatyer and N. Weiner, Phys. Rev. D 79, 015014 (2009) [arXiv:0810.0713 [hep-ph]].

[98] S. Cassel, J. Phys. G 37, 105009 (2010) [arXiv:0903.5307 [hep-ph]].

[99] J. L. Feng, M. Kaplinghat and H. -B. Yu, Phys. Rev. D 82, 083525 (2010) [arXiv:1005.4678 [hep-ph]].

[100] J. Beringer et al. [Particle Data Group Collaboration], Phys. Rev. D 86, 010001 (2012).

[101] R. J. Scherrer and M. S. Turner, Phys. Rev. D 33, 1585 (1986) [Erratum-ibid. D 34, 3263 (1986)].

[102] G. Steigman, B. Dasgupta and J. F. Beacom, Phys. Rev. D 86, 023506 (2012) [arXiv:1204.3622 [hep-ph]].

[103] J. B. Dent, S. Dutta and R. J. Scherrer, Phys. Lett. B 687, 275 (2010) [arXiv:0909.4128 [astro-ph.CO]].

[104] J. Chen and Y. -F. Zhou, JCAP 1304, 017 (2013) [arXiv:1301.5778 [hep-ph]].

[105] E. Aprile [XENON1T Collaboration], arXiv:1206.6288 [astro-ph.IM].

[106] J. Lavalle, Phys. Rev. D 82, 081302 (2010) [arXiv:1007.5253 [astro-ph.HE]].

[107] C. Evoli, I. Cholis, D. Grasso, L. Maccione and P. Ullio, Phys. Rev. D 85, 123511 (2012) [arXiv:1108.0664 [astro-ph.HE]].

[108] R. Kappl and M. W. Winkler, Phys. Rev. D 85, 123522 (2012) [arXiv:1110.4376 [hep-ph]].

[109] D. S. Akerib et al. [LUX Collaboration], Nucl. Instrum. Meth. A 704, 111 (2013) [arXiv:1211.3788 [physics.ins-det]].

[110] D. J. Koskinen, Mod. Phys. Lett. A 26, 2899 (2011).

[111] M. J. Strassler and K. M. Zurek, Phys. Lett. B 651, 374 (2007) [hep-ph/0604261].

[112] M. Heikinheimo, A. Racioppi, M. Raidal, C. Spethmann and K. Tuominen, arXiv:1305.4182 [hep-ph]. 